\title{
Introduction of a classical level in Quantum Theory
}

\section{Continuous monitoring}

\section{G. M. Prosperi}

the date of receipt and acceptance should be inserted later

\begin{abstract}
In an old paper of our group in Milano a formalism was introduced for the continuous monitoring of a system during a certain interval of time in the framework of a somewhat generalized approach to Q. M. The outcome was a distribution of probability on the space of all the possible continuous histories of a set of quantities to be considered as a kind of coarse grained approximation to some ordinary quantum observables commuting or not. In fact the main aim was the introduction of a classical level in the context of Quantum Mechanics, treating formally a set of basic quantities, to be considered as beables in the sense of Bell, as continuously taken under observation. However the effect of such assumption was a permanent modification of the Liouville-von Neumann equation for the statistical operator by the introduction of a dissipative term which is in conflict with basic conservation rules in all reasonable models we had considered. Difficulties were even encountered for a relativistic extension of the formalism. In this paper I propose a modified version of the original formalism which seems to overcome both difficulties. First I study the simple models of an harmonic oscillator and a free scalar field in which a coarse grain position and a coarse grained field respectively are treated as beables. Then I consider the more realistic case of Spinor Electrodynamics in which only certain coarse grained electric and magnetic fields and are introduced as classical variables no matter related quantities.
\end{abstract}

Keywords continuous monitoring · quantum mechanics · classical variables · spinor electrodynamics

PACS 03.65.Ta $\cdot 03.70 .+\mathrm{k}$

G. M. Prosperi

Dipartimento di Fisica dell'Universitá di Milano

I. N. F. N. , sezione di Milano

Via Celoria 16, I20133 Milano, (Italy)

E-mail: prosperi@mi.infn.it 


\section{Introduction}

In reference [1] a general formalism is introduced for the treatment of the continuous monitoring of a quantity or a set of quantities in the framework of Quantum Mechanics. The formalism turns out to be strictly related to a more particular one previously proposed by E. B. Davies for the observation of the counting times on a system of counters [2]. It is introduced in the context of the generalized formulation of Quantum Mechanics (GQM), based on the concept of positive operator valued measures (p.o.m.) and operation valued measures or instruments [2]-[4], in which approximate observations of non commuting observables and even sequences of observations of the same observables can be put on the same foot as the supposed exact observations considered in ordinary formulation (see app. A). The outcome is a distribution of probability on the set of all the possible continuous histories of the monitored quantities in the considered time interval. The class of event subsets is characterized in terms of time averages of the monitored quantities of the type

$$
a_{h}^{s}(t)=\int d t^{\prime} h\left(t-t^{\prime}\right) a^{s}\left(t^{\prime}\right)
$$

the $h(t)$ s being appropriate weight functions.

Later, various alternative formulations of the theory have been given and various aspects developed with interesting applications, particularly in the field of Quantum Information and Optics (for a recent presentation see e. g. [6] and references therein).

The original purpose of [1] was, however, to obtain a modification of ordinary Quantum Theory, in which an intrinsic classical level for some basic macroscopic quantities could be introduced, to solve consistently the problem of the interpretation of the theory in the sense of Bohr and of Von Neumann (which requires, as well known, the setup and output of an experiment to be described classically). The idea was that certain basic quantities should be chosen once for ever by an additional postulate and they should be thought as having at any time well defined values, considered as beables in the sense of Bell, by treating them formally as continuously observed. Then any observation on a microscopic system should be expressed in terms of the modifications that its interaction with the remaining part of the world produces in the value of such basic macroscopic quantities. Obviously the theory remains statistic and to any possible evolution of the basic quantities a precise probability is assigned, but interference among different histories are in principle excluded. Note that, even if in a completely different mathematical framework, similar conceptual ideas seem to be at the basis of the so called theory of the consistent histories [7], with reference to which see however ref. [8].

A significant property of the theory is that integrating over all possible histories of the basic quantities in a given time interval $\left(t_{0}, t_{F}\right)$ is equivalent in Schroedinger picture to introduce a dissipative term in the Liouville-Von Neumann evolution equation for the statistical operator. Alternatively in Heisenberg picture (which we find more convenient in this paper) this corresponds to 
ascribe a time dependence to the statistical operator according to an equation involving the same term. In the cases of our interest we can write

$$
\frac{\partial \hat{\rho}(t)}{\partial t}=-\sum_{j} \frac{\alpha_{j}}{4}\left[\hat{A}^{j}(t),\left[\hat{A}^{j}(t), \hat{\rho}(t)\right]\right] .
$$

Here $\hat{A}^{1}(t)=e^{i \hat{H} t} \hat{A}^{1} e^{-i \hat{H} t}, \hat{A}^{2}(t)=e^{i \hat{H} t} \hat{A}^{2} e^{-i \hat{H} t}, \ldots$ denote Heisenberg picture Hermitian operators not necessarily each other commuting and $\alpha_{1}, \alpha_{2}, \ldots$ certain positive constants. In the case, the monitored quantities $a^{1}, a^{2}, \ldots$ may be considered as a simultaneous coarse grained approximations of the ordinary quantum observables $A^{1}, A^{2}, \ldots$ with $\left\langle a^{s}(t)\right\rangle=\left\langle\hat{A}^{s}(t)\right\rangle=\operatorname{Tr}\left[\hat{A}^{s}(t) \rho(t)\right]$ and the variance $\left\langle\left(a_{h}^{s}(t)-\left\langle a_{h}^{s}(t)\right\rangle\right)^{2}\right\rangle$ (which is supposed to be small) expressed as the sum of an intrinsic classical term independent of $\hat{\rho}$ and a minor modification of the ordinary quantum variance for $A^{s}(t)$. To $a^{1}, a^{2}, \ldots$ in the following we shall conventionally refer as the macroscopic $A^{1}, A^{2}, \ldots{ }^{1}$

Notice that eq. (2) is trace and positivity preserving. The time dependence of $\hat{\rho}$ expresses the permanent effect of the modification introduced in the theory (formally the perturbation produced by the continuous monitoring), even when any information on the mentioned basic quantities is completely disregarded. The equation makes our theory in contact with theories that introduce ad hoc dissipative terms, as a noise, to simulate the interaction of the apparatus with an environment (see in particular in this connection ref. [9]) or theories that want introduce an intrinsic progressive decoherence and a spontaneous collapse at a more fundamental level. Theories of the latter type received considerable attention in the last thirty years (see e. g. [10] for general revues and complete references; a small representative sample, corresponding to various point of view, is reported in refs. [11]-[14]). However in our perspective interference terms among macroscopic states do not decay but do not exist in principle.

Unfortunately in the original form our theory, like all variants of decoherence and spontaneous collapse theories, meets two main difficulties: i) it is in conflict with basic conservation rules, specifically with energy conservation; ii) it does not seem it can be extended to relativistic field theories [1],[15]. ${ }^{2}$

Both these difficulties seem to be related to the requirement $\alpha_{1}, \alpha_{2}, \ldots$ to be positive. In this paper I show that it is possible to release such requirement and significant models can be constructed in which, by an appropriate choice of the operators $\hat{A}^{1}, \hat{A}^{2}, \ldots$, of the constants $\alpha_{1}, \alpha_{2}, \ldots$ and of the weight functions $h(t)$, a consistent positive probability distribution can be defined for the histories of the related basic quantities, the conservation rules respected and relativistic covariance achieved, when appropriate. This even if $\hat{\rho}(t)$ does not remain positive in the ordinary sense.

\footnotetext{
1 As a rule, across the paper we shall denote by hatted letter $\hat{A}, \hat{B}, \ldots$ operators on the Hilbert space of the system; by $A, B, \ldots$, the corresponding abstract observables, if they are self-adjoint; by the $a, b \ldots$ the related macroscopic quantities, if the case.

2 In ref. [16], in the somewhat different perspective of a finite difference Liouville von Neumann equation, the energy is conserved but the second difficulty remains
} 
First, to clarify the problem, we consider the two pedagogical models of the non relativistic harmonic oscillator and the real relativistic scalar field, both in the original and in the modified formalism, when we introduce as basic classical variables a macroscopic position and a macroscopic field respectively. Then we treat the physically more significant case of Spinor Quantum Electrodynamics with again the macroscopic components of the electromagnetic field assumed as classical or beables.

In the latter case eq. (2) has to be replaced by a Tomonaga-Schwinger like equation of the form

$$
\frac{\delta \hat{\rho}[\sigma]}{\delta \sigma(x)}=\frac{\gamma}{16}\left[\hat{F}_{\mu \nu}(x),\left[\hat{F}^{\mu \nu}(x), \hat{\rho}[\sigma]\right]\right],
$$

Note that the form of the right end side in this equation is practically completely determined by Lorentz and gauge invariance requirements but it corresponds to $\alpha_{1}, \alpha_{2}, \ldots$ not all positive. Naturally, as in any field theory the averages (1), in terms of which the probabilities are defined, have to be replaced by expressions of the type

$$
f_{h}^{\mu \nu}(x)=\int d^{4} x h\left(x-x^{\prime}\right) f^{\mu \nu}\left(x^{\prime}\right)
$$

and the restriction on the $h(x)$ s consists in the requirement that only time like wave vectors $k$ occur in the Fourier transform $\tilde{h}(k)$ or that they are dominant.

Note also that in the perspective of the model the dimensionless constant $\gamma$ which appears in eq. (3) should be understood as a new physical fundamental constant, that discriminates between classical and quantum scales.

Furthermore, as we told, only observables must be considered that can be expressed in terms of modifications of the macroscopic field. as a consequence, in comparison with the collapse models, our proposal corresponds to a specific choice of the "dissipative" term in the Liouville-von Newman equation, dropping the positivity requirement at the price of restricting the class of the observables.

The plan of the paper is the following one. In section 2 we review the original formalism of the continuous monitoring, manly to establish notations, and recall the important notion of functional generator. In sect 3 we consider the two pedagogical models of the harmonic oscillator and of the free real scalar case in the original and discuss the problem of the conservation rules. In sect. 4 we show, for the same models, how the formalism can be consistently modified in order to overcame the difficulties. In sect. 5 we introduce the mentioned more significant case of the spinor QED. Finally in sect. 6 we summarized the results and try to make some conclusions.

\section{Continuous monitoring of a set of quantities}

As well known, in the framework of the ordinary quantum mechanics, in which observables correspond to selfadjoint operators or equivalently to projection valued measures, a continuous monitoring of a quantity or of a set of 
quantities cannot be significantly considered due to a negative result usually recalled as Zeno's theorem or paradox [17]. According to such theorem, if we make repeated observations of the same quantity and let the time interval $\epsilon$ between two subsequent observations go to 0 , the value of the quantity is frozen to its initial value and does not longer change with time. In the Heisenberg picture the difficulty may be essentially related to the fact that operators corresponding to the same quantity at different times do not commute each others. On the contrary it can be understood that in Generalized Quantum Mechanics (GQM) it is possible to consider a double limit, in which as $\epsilon \rightarrow 0$, the observation in itself is made progressively less precise, in such way that a finite result is attained. The discussion in this section may be thought in this perspective, even if the double limit is not explicitly shown. The result is a generalized stochastic process in the sense of Gel'fand, in which a set of finite measure of histories of the quantities of interest has to be specified in terms of successive time averages of the type (1), rather than by values assumed at definite times.

In GMQ the case of a set of quantities continuously kept under observation can be introduced on complete analogy with the case of the observation of a set of quantities at a given time.

Let $\left(t_{0}, t_{F}\right)$ be a reference time interval (where $t_{F}$ might be eventually taken to $+\infty)$, let $\mathcal{Y}$ be the functional space of all possible histories $a(t) \equiv$ $\left(a^{1}(t), a^{2}(t), \ldots a^{p}(t)\right)$ of the quantities kept under observation and $\Sigma$ the class of the measurable subsets of $\mathcal{Y}$ according to some definition to be specified later. Let also denote by $\Sigma_{t_{a}}^{t_{b}} \subset \Sigma$ the class of the measurable subsets of $\mathcal{Y}$ corresponding to restrictions on the histories only in the interval $\left(t_{a}, t_{b}\right) \subset$ $\left(t_{0}, t_{F}\right)$. Then, we assume that to the continuous observation of $a(t)$ during the time interval $\left(t_{a}, t_{b}\right)$ an instrument, or normalized operation valued measure, $\mathcal{F}\left(t_{b}, t_{a} ; M\right)$ and the related p.o.m. $F\left(t_{b}, t_{a} ; M\right)=\mathcal{F}^{\prime}\left(t_{b}, t_{a} ; M\right) \hat{I}$ are associated with $M \in \Sigma_{t_{a}}^{t_{b}}$ and

$$
P\left(t_{b}, t_{a} ; M\right)=\operatorname{Tr}\left[\hat{F}\left(t_{b}, t_{a} ; M\right) \hat{\rho}\left(t_{a}\right)\right]=\operatorname{Tr}\left[\mathcal{F}\left(t_{b}, t_{a} ; M\right) \hat{\rho}\left(t_{a}\right)\right]
$$

interpreted as the probability of observing $a(t) \in M\left(\hat{\rho}\left(t_{a}\right)\right.$ being the statistical operator at the time $t_{a}$ ). According to the general definitions and axioms in GQM by an operation $\mathcal{F}$ we mean a positive mapping of the class of trace class operator in itself and by $\mathcal{F}^{\prime}$ its dual mapping on the class of the bounded operators according to the definition $\operatorname{Tr}[\hat{B} \mathcal{F} \hat{X}]=\operatorname{Tr}\left[\left(\mathcal{F}^{\prime} \hat{B}\right) \hat{X}\right]$. By normalized measure we intend that the mapping

$$
\mathcal{G}\left(t_{b}, t_{a}\right) \equiv \mathcal{F}\left(t_{b}, t_{a} ; \mathcal{Y}\right)
$$

is trace preserving and consequently $\hat{F}\left(t_{b}, t_{a} ; \mathcal{Y}\right)=\hat{I}$ and $P\left(t_{b}, t_{a} ; \mathcal{Y}\right)=1$.

Furthermore we assume also that $\mathcal{F}\left(t_{b}, t_{a} ; M\right)$ satisfies the Markovian relation

$$
\mathcal{F}\left(t_{c}, t_{b} ; N\right) \mathcal{F}\left(t_{b}, t_{a} ; M\right)=\mathcal{F}\left(t_{c}, t_{a} ; N \cap M\right),
$$

which expresses the independence of the observation of $a(t)$ in successive intervals of time (notice that $M \in \Sigma_{t_{a}}^{t_{b}}, N \in \Sigma_{t_{b}}^{t_{c}} \Rightarrow N \cap M \in \Sigma_{t_{a}}^{t_{c}}$ ). 
Then

$$
P\left(t_{c}, t_{a} ; N \cap M\right)=\operatorname{Tr}\left[\hat{F}\left(t_{c}, t_{b} ; N\right) \mathcal{F}\left(t_{b}, t_{a} ; M\right) \hat{\rho}\left(t_{a}\right)\right]
$$

gives the joint probability of observing $a(t) \in M$ in the time interval $\left(t_{a}, t_{b}\right)$ and $a(t) \in N$ in the interval $\left(t_{b}, t_{c}\right)$, while the ratio between (8) and (5) gives the conditional probability of observing $a(t) \in N$ if $a(t) \in M$ has been observed.

By setting $M=\mathcal{Y}$ in (8) we find

$$
P\left(t_{c}, t_{b} ; N\right)=P\left(t_{C}, t_{a} ; N \cup \mathcal{Y}\right)=\operatorname{Tr}\left[\hat{F}\left(t_{c}, t_{b} ; N\right) \mathcal{G}\left(t_{b}, t_{a}\right) \hat{\rho}\left(t_{a}\right)\right]
$$

Then, for comparison with (5), we can set $\hat{\rho}\left(t_{b}\right)=\mathcal{G}\left(t_{b}, t_{a}\right) \hat{\rho}\left(t_{a}\right)$ and so the perturbation produced on the system by its continuous observation introduces a kind of time dependence on the statistical operator in Heisenberg picture, that is described by the action of the mapping $\mathcal{G}\left(t_{b}, t_{a}\right)$

Finally, on analogy of the usual probability theory, we can define a characteristic functional operator (CFO) as the functional Fourier transform

$$
\mathcal{G}\left(t_{b}, t_{a} ;[\xi(t)]\right)=\int \mathcal{F}\left(t_{b}, t_{a} ; \mathcal{D}_{c} M\right) \exp \left\{-i \int_{t_{a}}^{t_{b}} d t \xi^{s}(t) a^{s}(t)\right\}
$$

where $\mathcal{D}_{\mathrm{c}} M$ denotes the measure of an elementary set in the functional space $\mathcal{Y}$ (the index "c" referring to the interpretation of $a(t)$ as a classical history, to distinguish the classical functional measure from the quantum path integral measure, which shall be used in the following). The concept of CFO turns out to be very useful, not only to study the properties of the structure we are considering but even to construct it.

Notice that in terms of $\mathcal{G}\left(t_{b}, t_{a} ;[\xi(t)]\right)$ assumption (7) becomes

$$
\mathcal{G}\left(t_{c}, t_{b} ;[\xi(t)]\right) \mathcal{G}\left(t_{b}, t_{a} ;[\xi(t)]\right)=\mathcal{G}\left(t_{c}, t_{a} ;[\xi(t)]\right) .
$$

Then, if we set

$$
\mathcal{G}(t+d t, t ;[\xi(t)])=1+\mathcal{K}(t ; \xi(t)) d t,
$$

we can write the differential equation

$$
\frac{\partial}{\partial t} \mathcal{G}\left(t, t_{a} ;[\xi]\right)=\mathcal{K}(t ; \xi(t)) \mathcal{G}\left(t, t_{a} ;[\xi]\right)
$$

This can be formally solved as

$$
\left.\mathcal{G}\left(t_{b}, t_{a} ; \xi\right]\right)=\mathrm{T} \exp \int_{t_{a}}^{t_{b}} d t \mathcal{K}(t ; \xi(t))
$$

$\mathrm{T}$ being the usual time ordering prescription.

Now let us observe that

$$
\mathcal{G}\left(t_{b}, t_{a} ;[0]\right)=\mathcal{F}\left(t_{b}, t_{a} ; \mathcal{Y}\right) \equiv \mathcal{G}\left(t_{b}, t_{a}\right)
$$

Then, setting $\xi(t)=0$ in (11-14), there follow the semigroup property

$$
\mathcal{G}\left(t_{c}, t_{a}\right)=\mathcal{G}\left(t_{c}, t_{b}\right) \mathcal{G}\left(t_{b}, t_{a}\right),
$$


the equation

$$
\frac{\partial}{\partial t} \mathcal{G}\left(t, t_{a}\right)=\mathcal{L}(t) \mathcal{G}\left(t, t_{a}\right)
$$

and also

$$
\mathcal{G}\left(t_{b}, t_{a}\right)=\mathrm{T} \exp \int_{t_{a}}^{t_{b}} d t \mathcal{L}(t)
$$

where obviously

$$
\mathcal{L}(t)=\mathcal{K}(t ; 0)
$$

and, being $\mathcal{G}\left(t_{b}, t_{a}\right)$ trace preserving,

$$
\operatorname{Tr}\{\mathcal{L}(t) \hat{\rho}\}=0 .
$$

Under some additional restrictions eq. (20) and the requirement of positivity (actually of complete positivity) implies $\mathcal{L}(t)$ to be of the general form

$$
\mathcal{L}(t) \hat{\rho}=-\sum_{s=1}^{p} \alpha_{s}\left(\hat{R}^{s \dagger} \hat{R}^{s} \hat{\rho}+\hat{\rho} \hat{R}^{s^{\dagger}} \hat{R}^{s}-2 \hat{R}^{s} \hat{\rho} \hat{R}^{s \dagger}\right) .
$$

$\alpha_{s}$ being appropriate positive constants (cf. [18]). Note that eq. (2) is a particular case of eq. (21) for $\hat{R}^{s}=\frac{1}{2} \hat{A}^{s}$.

Conversely we can set

$$
\mathcal{F}\left(t_{b}, t_{a} ; \mathcal{D}_{\mathrm{c}} M\right)=\mathbf{f}\left(t_{b}, t_{a} ;[a(t)]\right) \mathcal{D}_{\mathrm{c}} M,
$$

with

$$
\begin{aligned}
& \mathbf{f}\left(t_{b}, t_{a} ;[a(t)]\right)= \\
& =\int \mathcal{D}_{c} \xi \exp \left\{i \sum_{s=1}^{p} \int_{t_{a}}^{t_{b}} d t \xi^{s}(t) a^{s}(t)\right\} \mathcal{G}\left(t_{b}, t_{a} ;[\xi(t)]\right.
\end{aligned}
$$

and define the probability density

$$
p\left(t_{b}, t_{a} ;[a(t)]\right)=\operatorname{Tr}\left\{\mathbf{f}\left(t_{b}, t_{a} ;[a(t)]\right) \rho\left(t_{a}\right)\right\} .
$$

Here the measure $\mathcal{D}_{\mathrm{c}} \xi$ is normalized in such a way that

$$
\int \mathcal{D}_{\mathrm{c}} \xi \exp \left\{-i \sum_{s=1}^{p} \int_{t_{a}}^{t_{b}} d t \xi^{s}(t)\left(a^{s}(t)-a^{\prime s}(t)\right)\right\}=\delta\left([a(t)]-\left[a^{\prime}(t)\right]\right),
$$

$\delta\left([a(t)]-\left[a^{\prime}(t)\right]\right)$ being the $\delta$ - functional with respect to the measure $\mathcal{D}_{\mathrm{c}} a$.

Formally, this may be achieved assuming the interval $\left(t_{a}, t_{b}\right)$ divided in $N$ equal parts of amplitude $\epsilon=\left(t_{b}-t_{a}\right) / N$ and defining

$$
\mathcal{D}_{\mathrm{c}} M \equiv \mathcal{D}_{\mathrm{c}} a=\left(\frac{\epsilon}{2 \pi}\right)^{N p / 2} d^{p} a_{1} \ldots d^{p} a_{N}, \quad \mathcal{D}_{\mathrm{c}} \xi=\left(\frac{\epsilon}{2 \pi}\right)^{N p / 2} d^{p} \xi_{1} \ldots d^{p} \xi_{N},
$$

and

$$
\delta\left([a(t)]-\left[a^{\prime}(t)\right]\right)=\left(\frac{2 \pi}{\epsilon}\right)^{N p / 2} \delta^{p}\left(a_{1}-a_{2}^{\prime}\right) \ldots \delta\left(a_{N}-a_{n}^{\prime}\right),
$$


to be understood in the limit $N \rightarrow \infty$.

Eqs. (22) and (23) enable us to reconstruct $\mathcal{F}\left(t_{b}, t_{a} ; M\right)$ given $\mathcal{G}\left(t_{b}, t_{a} ;[\xi]\right)$ or $\mathcal{K}(t, \xi(t))$. Naturally, $\mathcal{K}(t, \xi(t))$ has to be of an appropriate form in order $\mathbf{f}\left(t_{1}, t_{0} ;[a(t)]\right)$, as defined by $(23)$, to be completely positive and definition $(24)$ sensible.

Two such forms are known: the Gaussian form and the Poissonian form, and obviously a sum of the two. We are interested only in the first form which consists in setting:

$$
\mathcal{K}(t, \xi(t)) \hat{\rho}=\mathcal{L}(t) \hat{\rho}-i \sum_{s=1}^{p} \xi^{s}(t)\left(\hat{R}^{s}(t) \hat{\rho}+\hat{\rho} \hat{R}^{s^{\dagger}}(t)\right)-\sum_{s=1}^{p} \frac{1}{4 \alpha_{s}} \xi^{s}(t) \hat{\rho}
$$

That (28) is a correct prescription can be shown in a variety of ways, by path integral, operator or stochastic calculus techniques. The point is to obtain a more explicit expression for the right end side of eq. (14) and replace it in (23) showing that this can be written in the form

$$
\mathbf{f}\left(t_{b}, t_{a} ;[a(t)]\right)=\hat{\Gamma}\left(t_{b}, t_{a}\right) \hat{\rho}\left(t_{a}\right) \hat{\Gamma}^{\dagger}\left(t_{b}, t_{a}\right) .
$$

In the following we shall use path integral techniques.

Notice that from (23), (25) we have

$$
\int \mathcal{D}_{\mathrm{c}} a \mathbf{f}\left(t_{b}, t_{a} ;[a(t)]\right)=\int \mathcal{D}_{\mathrm{c}} \xi \delta([\xi]) \mathcal{G}\left(t_{b}, t_{a} ;[\xi]\right)=\mathcal{G}\left(t_{b}, t_{a}\right)
$$

and so

$$
\int \mathcal{D}_{\mathrm{c}} a \operatorname{Tr}\left\{\mathbf{f}\left(t_{b}, t_{a} ;[a(t)]\right) \hat{\rho}\left(t_{a}\right)\right\}=\operatorname{Tr} \hat{\rho}\left(t_{a}\right)=1
$$

Likewise, for the momenta of the components of $a(t)$ at certain definite times $t_{1}, t_{2}, \ldots t_{N}$ in the interval $\left(t_{a}, t_{b}\right)$ we obtain

$$
\begin{aligned}
& \left\langle a^{s_{1}}\left(t_{1}\right) a^{s_{2}}\left(t_{2}\right) \ldots a^{s_{l}}\left(t_{l}\right)\right\rangle=\int D_{\mathrm{c}} a a^{s_{1}}\left(t_{1}\right) a^{s_{2}}\left(t_{2}\right) \ldots a^{s_{l}}\left(t_{l}\right) \\
& \operatorname{Tr}\left\{\mathbf{f}\left(t_{b}, t_{a} ;[a(t)]\right) \hat{\rho}\left(t_{a}\right)\right\}=\left.i^{l} \operatorname{Tr}\left\{\frac{\delta}{\delta \xi^{s_{1}}\left(t_{1}\right)} \ldots \frac{\delta}{\delta \xi^{s_{l}}\left(t_{l}\right)} \mathcal{G}\left(t_{b}, t_{a} ;[\xi]\right) \hat{\rho}\left(t_{a}\right)\right\}\right|_{\xi=0} .
\end{aligned}
$$

and in particular for the expectation value of a single component

$$
\begin{aligned}
& \left\langle a^{s}(t)\right\rangle=\operatorname{Tr}\left[\left.i \frac{\delta}{\delta \xi^{s}(t)} \mathcal{G}\left(t_{b}, t_{a} ;[\xi]\right)\right|_{\xi=0} \hat{\rho}\left(t_{a}\right)\right]= \\
& =\operatorname{Tr}\left[\left.\mathcal{G}\left(t_{b}, t\right) i \frac{\partial}{\partial \xi^{s}} \mathcal{K}(t, \xi)\right|_{\xi=0} \mathcal{G}\left(t, t_{a}\right) \hat{\rho}\left(t_{a}\right)\right]=\operatorname{Tr}\left[\hat{A}^{s} \mathcal{G}\left(t, t_{a}\right) \hat{\rho}\left(t_{a}\right)\right]
\end{aligned}
$$

with

$$
\hat{A}^{s}(t)=\hat{R}^{s}(t)+\hat{R}^{s \dagger}(t) .
$$

Therefore we can talk of $a^{1}(t), a^{2}(t), \ldots, a^{p}(t)$ as the value at time $t$ of the macroscopic or, in our interpretation, the classical counterpart of the 
quantum observable associated to $\hat{A}^{s}$ even if $A^{1}, A^{2}, \ldots, A^{p}$ do not each others commute.

For the second momenta we have

$$
\begin{gathered}
\left\langle a^{s}(t) a^{s^{\prime}}\left(t^{\prime}\right)\right\rangle=\left.\delta\left(t-t^{\prime}\right) \operatorname{Tr}\left\{\frac{\partial^{2} \mathcal{K}(t, \xi)}{\partial \xi^{s} \partial \xi^{s^{\prime}}} \mathcal{G}\left(t, t_{a}\right) \hat{\rho}\right\}\right|_{\xi=0}- \\
-\left.\theta\left(t-t^{\prime}\right) \operatorname{Tr}\left\{\frac{\partial K(t, \xi)}{\partial \xi^{s}} \mathcal{G}\left(t, t^{\prime}\right) \frac{\partial K\left(t^{\prime}, \xi\right)}{\partial \xi^{s^{\prime}}} \mathcal{G}\left(t^{\prime}, t_{a}\right) \hat{\rho}\right\}\right|_{\xi=0}- \\
-\left.\theta\left(t^{\prime}-t\right) \operatorname{Tr}\left\{\frac{\partial K\left(t^{\prime}, \xi\right)}{\partial \xi^{s^{\prime}}} \mathcal{G}\left(t^{\prime}, t\right) \frac{\partial K(t, \xi)}{\partial \xi^{s}} \mathcal{G}\left(t, t_{a}\right) \hat{\rho}\right\}\right|_{\xi=0} \cdot
\end{gathered}
$$

The occurrence of the $\delta$ term in eq. (35) shows that only time averages of the type

$$
a_{h}=\int d t h(t) \cdot a(t) \equiv \int d t \sum_{s=1}^{p} h^{s}(t) a^{s}(t),
$$

are actually significant, the weight functions $h(t) \equiv\left(h^{1}(t), \ldots, h^{p}(t)\right)$ being elements of the dual space $\mathcal{Y}^{\prime}$. Therefore the class $\Sigma$ of the measurable set in $\mathcal{Y}$ should be defined in terms of such quantities and we can talk of the statistics of the histories as of a generalized stochastic process in the sense of Gel'fand.

Typically one can choose $\mathcal{Y}=\mathcal{E}^{\prime} \times \mathcal{E}^{\prime} \times \ldots \mathcal{E}^{\prime}$ and $\mathcal{Y}^{\prime}=\mathcal{E} \times \mathcal{E} \times \ldots \mathcal{E}$, where $\mathcal{E}$ is the class of the function with compact support in the $t$-axis and infinitely differentiable everywhere with the possible exception of finite discontinuities on the border of the support and $\mathcal{E}^{\prime}$ the dual space of $\mathcal{E}$ (a subset of the Schwartz distribution space; punctual spectrum is not allowed). The possibility of finite discontinuities on the border has to be admitted to give full meaning to eq.(11).

Furthermore we recall that, if $h_{1}(t), h_{2}(t), \ldots h_{l}(t)$ are a set of elements of $\mathcal{Y}^{\prime}$ as above and $B$ a Borel set in $\mathbb{R}^{n}$, the subset of $\mathcal{Y}$

$$
C\left(h_{1}, h_{2}, \ldots h_{n} ; B\right)=\left\{a(t) \in \mathcal{Y} ;\left(a_{h_{1}}, a_{h_{1}} \ldots a_{h_{1}}\right) \in B\right\}
$$

is called a cylinder set. Then $\Sigma$ can be identified with the $\sigma$-algebra generated by all the cylinder sets for any choice of $n, B \subset \mathbb{R}^{n}$ and of $h_{1}, h_{2}, \ldots h_{n}$. The sub-algebra $\Sigma_{t_{a}}^{t_{b}}$ is the same, but with $h_{j}$ with support in the interval $\left(t_{a}, t_{b}\right)$.. Alternatively, we may refer to densities of probability of the form

$$
\begin{aligned}
& p\left(\bar{a}_{1}, h_{1} ; \bar{a}_{2}, h_{2} ; \ldots \bar{a}_{l}, h_{l}\right)= \\
& =\int \mathcal{D}_{\mathrm{c}} a \delta\left(\bar{a}_{1}-a_{h_{1}}\right) \ldots \delta\left(\bar{a}_{l}-a_{h_{l}}\right) \operatorname{Tr}\left\{\mathbf{f}\left(t_{b}, t_{a} ;[a]\right) \hat{\rho}\right\}=\frac{1}{(2 \pi)^{l}} \int d k_{1} \ldots \\
& \ldots d k_{l} e^{i\left(k_{1} \bar{a}_{1}+\ldots k_{l} \bar{a}_{l}\right)} \int \mathcal{D}_{\mathrm{c}} a e^{-i\left(k_{1} a_{h_{1}}+\ldots k_{l} a_{h_{l}}\right)} \operatorname{Tr}\left\{\mathbf{f}\left(t_{b}, t_{a} ;[a]\right) \hat{\rho}\right\}= \\
& =\frac{1}{(2 \pi)^{l}} \int d k_{1} \ldots d k_{l} e^{i\left(k_{1} \bar{a}_{1}+n \ldots k_{l} \bar{a}_{l}\right)} \operatorname{Tr}\left\{\mathcal{G}\left(t_{b}, t_{a} ;\left[k_{1} h_{1}\right)+\ldots k_{l} h_{l}\right] \hat{\rho}\right\}
\end{aligned}
$$

where $h_{1}(t), h_{2}(t), \ldots h_{l}(t)$ are independent elements of $\mathcal{Y}$ with support in $\left(t_{a}, t_{b}\right)$. A sensible choice could be

$$
h_{j}(t)=\left(n_{j}^{1} h\left(t-t_{j}\right), n_{j}^{2} h\left(t-t_{j}\right), \ldots n_{j}^{p} h\left(t-t_{j}\right)\right),
$$


$h(t)$ being a function different from zero only in a narrow neighboring of $t=0$ such that $\int d t h(t)=1 ; n_{j}$ for $j=1,2, \ldots l$ unitary vectors in the euclidean $p$ dimensional space $\mathbb{R}^{p} ; t_{1}, t_{2}, \ldots t_{l}$ certain intermediate times between $t_{a}$ and $t_{b}$.

\section{Two pedagogical models}

In view of our interpretation, let us now consider two specific pedagogical models, the case of the non relativistic harmonic oscillator and of the relativistic scalar field in which the quantities treated as continuously monitored are a macroscopic position $q(t)$ and a macroscopic field $\varphi(x)$ respectively.

In the first case, with Lagrangian and Hamiltonian

$$
L=\frac{1}{2}\left(\dot{Q}^{2}-\omega^{2} Q^{2}\right) \quad \text { and } \quad H=\frac{1}{2}\left(P^{2}+\omega^{2} Q^{2}\right)
$$

respectively, we can assume

$$
\mathcal{L}(t) \hat{\rho}=-\frac{\alpha}{4}[\hat{Q},[\hat{Q}, \hat{\rho}]]
$$

with $\alpha$ a positive constant with dimensions $t^{-2}$, and

$$
\mathcal{K}(t ; \xi(t)) \hat{\rho}=-\frac{\alpha}{4}[\hat{Q},[\hat{Q}, \hat{\rho}]]-\frac{i}{2} \xi(t)\{\hat{Q}, \hat{\rho}\}-\frac{1}{4 \alpha} \xi^{2}(t),
$$

corresponding to have set in eq. (21) and (28) $p=1$ and $\hat{R}=\frac{1}{2} \hat{Q}$.

Notice that eqs. (33) and (35) become

$$
\langle q(t)\rangle=\langle\hat{Q}(t)\rangle_{\mathrm{QM}} \equiv \operatorname{Tr}\left\{\hat{Q}(t) \mathcal{G}\left(t, t_{0}\right) \hat{\rho}_{0}\right\}
$$

and

$$
\begin{aligned}
\left\langle q(t) q\left(t^{\prime}\right)\right\rangle & =\frac{1}{2 \alpha} \delta\left(t-t^{\prime}\right) \\
+ & \theta\left(t-t^{\prime}\right) \frac{1}{2} \operatorname{Tr}\left(\hat{Q}(t) \mathcal{G}\left(t, t^{\prime}\right)\left\{\hat{Q}\left(t^{\prime}\right), \mathcal{G}\left(t^{\prime}, t_{0}\right) \hat{\rho}_{0}\right\}\right) \\
+ & \theta\left(t^{\prime}-t\right) \frac{1}{2} \operatorname{Tr}\left(\hat{Q}\left(t^{\prime}\right) \mathcal{G}\left(t^{\prime}, t\right)\left\{\hat{Q}(t), \mathcal{G}\left(t, t_{0}\right) \hat{\rho}_{0}\right\}\right) .
\end{aligned}
$$

According to (36) we also set

$$
q_{h}(t)=\int d t^{\prime} h\left(t-t^{\prime}\right) q\left(t^{\prime}\right) \quad \text { and } \quad \hat{Q}_{h}(t)=\int d t^{\prime} h\left(t-t^{\prime}\right) \hat{Q}\left(t^{\prime}\right)
$$

where convenient choices for $h(t)$ could be

$$
h(t)=\frac{1}{\tau} \chi_{\left(-\frac{\tau}{2}, \frac{\tau}{2}\right)}(t), \quad \frac{1}{\tau \sqrt{\pi}} e^{-\frac{t^{2}}{\tau^{2}}}, \quad \frac{1}{\pi} \frac{\sin \frac{t}{\tau}}{t}
$$


$\left[\chi_{\left(-\frac{\tau}{2}, \frac{\tau}{2}\right)}(t)\right.$ being the characteristic function of the interval $\left.\left(-\frac{\tau}{2}, \frac{\tau}{2}\right)\right]$, even if, strictly speaking, only the first has compact support.

Then obviously we have

$$
\left\langle q_{h}(t)\right\rangle=\left\langle\hat{Q}_{h}(t)\right\rangle_{\mathrm{QM}}
$$

and, if $\tau$ is so small that $\mathcal{G}\left(t, t^{\prime}\right)$ can be replaced in (44) by the identity,

$$
\left\langle\left(q_{h}(t)-\left\langle q_{h}(t)\right\rangle\right)^{2}\right\rangle=\frac{1}{2 \alpha \tau}+\left\langle\left(\hat{Q}_{h}(t)-\left\langle\hat{Q}_{h}(t)\right\rangle_{\mathrm{QM}}\right)^{2}\right\rangle_{\mathrm{QM}},
$$

where the first term has no counterpart in ordinary quantum theory and it is what we have called the intrinsic classical part of the variance in the introduction.

Coming to the second model, to extend the formalism to fields and make the theory relativistic covariant, eq. (13) has to be replaced by

$$
\frac{\delta}{\delta \sigma(x)} \mathcal{G}\left(\sigma, \sigma_{0} ;[j(x)]\right)=\mathcal{K}(x, j(x)) \mathcal{G}\left(\sigma, \sigma_{0} ;[j(x)]\right),
$$

where $x \equiv(t, \mathbf{x}), \sigma$ and $\sigma_{0}$ are space-like hypersurfaces and $\mathcal{K}(x, j(x))$ is expressed only in terms of field operators at the point $x$. From this equation it follows in any arbitrary reference

$$
\frac{\partial}{\partial t} \mathcal{G}\left(t, t_{0} ;[j]\right)=\int d^{3} \mathbf{x} \mathcal{K}(t, \mathbf{x} ; j(x)) \mathcal{G}\left(t, t_{0} ;[j]\right),
$$

which is of the general form (13), with $\mathbf{x}$ playing the role of a component index (cf. eq. (28).

Note that, in order eq. (49) to be consistent, the following condition has to be satisfied on any space-like $\sigma$

$$
\mathcal{K}(x, j(x)) \mathcal{K}\left(x^{\prime}, j\left(x^{\prime}\right)\right)=\mathcal{K}\left(x^{\prime}, j\left(x^{\prime}\right)\right) \mathcal{K}(x, j(x)) .
$$

In the case of the free real scalar field with density of Lagrangian

$$
L(x)=\frac{1}{2}\left(\partial_{\mu} \varphi(x) \partial^{\mu} \varphi(x)-m^{2} \varphi^{2}(x)\right)
$$

and the energy-momentum tensor

$$
T_{\mu \nu}(x)=\partial_{\mu} \varphi(x) \partial_{\nu} \varphi(x)-g_{\mu \nu} L(x),
$$

we can set

$$
\mathcal{L}(x) \hat{\rho}=-\frac{\alpha}{4}[\hat{\varphi}(x),[\hat{\varphi}(x), \hat{\rho}]]
$$

and

$$
\mathcal{K}(x, j(x)) \hat{\rho}=\mathcal{L}(x) \hat{\rho}-\frac{i}{2} j(x)\{\hat{\varphi}(x), \hat{\rho}\}-\frac{1}{4 \alpha} j^{2}(x) \hat{\rho} .
$$

Note that then eq. (51) is satisfied as consequence of $\left[\hat{\varphi}(x), \hat{\varphi}\left(x^{\prime}\right)\right]=0$ for $x^{\prime}$ out of the light cone of $x$. 
Obviously (36) become space-time averages of the type

$$
\phi_{h}(x)=\int d^{4} x^{\prime} h\left(x^{\prime}-x\right) \phi\left(x^{\prime}\right) \quad \text { and } \quad \hat{\varphi}_{h}(x)=\int d^{4} x^{\prime} h\left(x^{\prime}-x\right) \hat{\varphi}\left(x^{\prime}\right),
$$

where e. g. one could chose

$$
h(x)=\frac{1}{\pi^{2} \tau a^{3}} e^{-\frac{t^{2}}{\tau^{2}}-\frac{\mathbf{x}^{2}}{a^{2}}} .
$$

As we mentioned, however, equations of the type (41) and (54) are in conflict with the energy conservation (meaning with such term in the present context the conservation of $\langle\hat{H}\rangle_{\mathrm{QM}}$ and $\left.\left\langle\int d^{3} \mathbf{x} \hat{T}_{00}(x)\right\rangle_{\mathrm{QM}}\right)$.

In fact for any ordinary observable $\hat{A}(t)$ we have

$$
\begin{aligned}
& \frac{d}{d t}\langle\hat{A}(t)\rangle_{\mathrm{QM}}=\operatorname{Tr}\left[\frac{d \hat{A}}{d t} \mathcal{G}\left(t, t_{0}\right) \hat{\rho}\right]+\operatorname{Tr}\left[\hat{A}(t) \frac{\partial}{\partial t} \mathcal{G}\left(t, t_{0}\right) \hat{\rho}\right]= \\
& =\left\langle\frac{d \hat{A}(t)}{d t}+\mathcal{L}^{\prime}(t) \hat{A}(t)\right\rangle_{\mathrm{QM}},
\end{aligned}
$$

where again by $\mathcal{L}^{\prime}$ we denote the dual mapping of $\mathcal{L}$.

For the harmonic oscillator, since obviously $\frac{d}{d t} \hat{H}=0$, we have from canonical commutation rules

$$
\frac{d}{d t}\langle\hat{H}\rangle_{\mathrm{QM}}=\left\langle\mathcal{L}^{\prime}(t) \hat{H}\right\rangle_{\mathrm{QM}}=-\frac{\alpha}{4}[[\hat{H}(t), \hat{Q}(t)], \hat{Q}(t)]=\frac{\alpha}{4} \neq 0 .
$$

y) and so

For the real scalar field, in a similar way, $\left.\mathcal{L}^{\prime}(t, \mathbf{y}) \hat{T}(t, \mathbf{x})\right)=-g^{0 \nu} \delta^{3}(\mathbf{x}-$

$$
\begin{aligned}
& \partial_{\mu}\left\langle\hat{T}^{\mu \nu}(t, \mathbf{x})\right\rangle_{\mathrm{QM}}= \\
& =\left\langle\partial_{\mu} \hat{T}^{\mu \nu}(t, \mathbf{x})+\int d^{3} \mathbf{y} \mathcal{L}^{\prime}(t, \mathbf{y}) \hat{T}^{0 \nu}(t, \mathbf{x})\right\rangle_{\mathrm{QM}}=\frac{1}{4} g^{0 \nu} \delta(\mathbf{0}) .
\end{aligned}
$$

The latter is not only different from 0 but even infinite and non-covariant, showing that $\mathcal{L}(x)$ as given by (54) is hill defined.

\section{Modified formalism}

At a formal level the above difficulties can be overcome modifying the definition of $\mathcal{L}(t)$ and $\mathcal{L}(x)$.

For the harmonic oscillator we can replace (41) with

$$
\begin{aligned}
\mathcal{L}(t)=- & \frac{\alpha}{4}\left([\hat{P}(t),[\hat{P}(t), \hat{\rho}]]-\omega^{2}[\hat{Q}(t),[\hat{Q}(t), \hat{\rho}]]\right) \\
& =-\frac{\alpha}{4}\left([\dot{\hat{Q}}(t),[\dot{\hat{Q}}(t), \hat{\rho}]]-\omega^{2}[\hat{Q}(t),[\hat{Q}(t), \hat{\rho}]]\right),
\end{aligned}
$$


$\alpha$ being now dimensionless. Then we have immediately

$$
\mathcal{L}^{\prime}(t) \hat{H}=0 \quad \Rightarrow \quad \frac{d}{d t}\langle\hat{H}\rangle_{\mathrm{QM}}=0 .
$$

Similarly for the scalar field we can take

$$
\mathcal{L}(x) \hat{\rho}=-\frac{\alpha}{4}\left(\left[\partial_{\mu} \hat{\varphi}(x),\left[\partial^{\mu} \hat{\varphi}(x), \hat{\rho}\right]\right]-m^{2}[\hat{\varphi}(x),[\hat{\varphi}(x), \hat{\rho}]]\right)
$$

and then again

$$
\mathcal{L}^{\prime}(t, \mathbf{y}) \hat{T}^{0 \nu}(t, \mathbf{x})=0 \quad \Rightarrow \quad \partial_{\mu}\left\langle\hat{T}^{\mu \nu}(x)\right\rangle_{\mathrm{QM}}=0 .
$$

Note the similarity between the expressions (61) and (63) and the corresponding Lagrangian or Lagrangian density (40) and (53) respectively.

Obviously assumptions (61) and (63) correspond to $\alpha_{j}$ of both signs in $(2)$ and the mappings $\mathcal{G}\left(t, t_{0}\right)$ and $\mathcal{G}\left(\sigma, \sigma_{0}\right)$ are no longer positive in themselves. However we shall show that it is possible to modify the definition of $\mathcal{K}(t,[\xi])$ and $\mathcal{K}(x,[j])$ in such a way that the densities of probability $p\left(t_{F}, t_{0} ;[q]\right)=$ $\operatorname{Tr}\left[\mathbf{f}\left(t_{F}, t_{0} ;[q]\right) \hat{\rho}_{0}\right]$ and $p\left(\sigma_{F}, \sigma_{0} ;[\phi]\right)=\operatorname{Tr}\left[\mathbf{f}\left(\sigma_{F}, \sigma_{0} ;[\phi]\right) \hat{\rho}_{0}\right]$ remain positive. Furthermore the procedure can be extended to the case of spinor electrodynamics, the quantity to be interpreted as classical being a macroscopic e. $\mathrm{m}$. field.

In the case harmonic oscillator we assume

$$
\begin{aligned}
\mathcal{K}(t,[\xi]) \hat{\rho} & =\mathcal{L}(t)-\frac{i}{2} \xi(t)\{\hat{Q}(t), \hat{\rho}\} \\
& -\frac{1}{2 \alpha} \int_{t_{0}}^{t} d t^{\prime} G\left(t-t^{\prime}\right) \xi(t) \xi\left(t^{\prime}\right) \hat{\rho}
\end{aligned}
$$

where

$$
G\left(t-t^{\prime}\right)=\frac{1}{T} \sum_{n=-\infty}^{\infty} \frac{1}{k_{n}^{2}-\omega^{2}} e^{i k_{n}\left(t-t^{\prime}\right)} \simeq \frac{1}{2 \pi} \int_{-\infty}^{\infty} d k \frac{1}{k^{2}-\omega^{2}} e^{i k\left(t-t^{\prime}\right)}
$$

$\left(T=t_{F}-t_{0}, \quad k_{n}=\frac{2 \pi n}{T}, \quad n=0, \pm 1, \pm 2, \ldots\right)$ is the solution of the equation

$$
\mathrm{K} G\left(t-t^{\prime}\right) \equiv-\left(\frac{d^{2}}{d t^{2}}+\omega^{2}\right) G\left(t-t^{\prime}\right)=\delta\left(t-t^{\prime}\right)
$$

i.e. it is the inverse of the differential operator K. Obviously in the last expression in (66) some kind of regularization should be understood for $k \sim \omega$, the precise prescription depending on the ratio between the total interval $T=t_{F}-t_{0}$ and the classic period of the oscillator $T_{P}=2 \pi / \omega\left(T / T_{P}\right.$ equal an half odd integer would correspond to the principal value prescription). However, this is essentially immaterial, due to our specific procedure and, in particular, the way the continuous $G\left(t-t^{\prime}\right)$ is approached from the lattice. 
We can also write

$$
\begin{aligned}
& K\left(t-t^{\prime}\right) \equiv \mathrm{K} \delta\left(t-t^{\prime}\right)= \\
& \quad=\frac{1}{T} \sum_{n=-\infty}^{\infty}\left(k_{n}^{2}-\omega^{2}\right) e^{i k_{n}\left(t-t^{\prime}\right)} \simeq \frac{1}{2 \pi} \int_{-\infty}^{\infty} d k\left(k^{2}-\omega^{2}\right) e^{i k\left(t-t^{\prime}\right)} .
\end{aligned}
$$

Note that, with our modified definition, $\mathcal{K}(t,[\xi])$ depends not only on the value of $\xi$ at the time $t$, as in (42), but on its entire history before $t$. We can again define $\left.\mathcal{G}\left(t_{b}, t_{a} ;[\xi]\right)\right)$ by $(14)$, but it has to be understood that the integral in $t^{\prime}$ is extended in any case from $t_{0}$ to $t$. Then eqs. (11) and (16) remain valid but, as we shall see, eq. (7) holds only in the limit in which the weight functions by which $M$ and $N$ are defined have sufficiently separate supports.

In the following it is convenient to make reference to the entire interval $\left(t_{0}, t_{F}\right)$. Then solving eq. (13) by standard path integral techniques (for the present purpose the perspective of [20] is particular convenient), we obtain

$$
\begin{aligned}
& \left\langle Q_{F}, t_{F}\left|\mathcal{G}\left(t_{F}, t_{0} ;[\xi]\right) \hat{\rho}_{0}\right| Q_{F}^{\prime}, t_{F}\right\rangle= \\
& =\int d Q_{0} \int d Q_{0}^{\prime}\left\langle Q_{0}, t_{0}\left|\hat{\rho}_{0}\right| Q^{\prime} t_{0}\right\rangle \int_{Q_{0}}^{Q_{F}} \mathcal{D} Q \int_{Q_{0}^{\prime}}^{Q_{F}^{\prime}} \mathcal{D} Q^{\prime} \\
& \exp \sum_{i=0}^{N-1}\left\{-\frac{\alpha}{4}\left[\frac{1}{\epsilon}\left(\left(Q_{i+1}-Q_{i}\right)-\left(Q_{i+1}^{\prime}-Q_{i}^{\prime}\right)\right)^{2}-\epsilon \omega^{2}\left(Q_{i}-Q_{i}^{\prime}\right)^{2}\right]\right. \\
& +\frac{i}{2}\left(\frac{1}{\epsilon}\left(Q_{i+1}-Q_{i}\right)^{2}-\epsilon \omega^{2} Q_{i}^{2}\right)-\frac{i}{2}\left(\frac{1}{\epsilon}\left(Q_{i+1}^{\prime}-Q_{i}^{\prime}\right)^{2}-\epsilon \omega^{2} Q_{i}^{\prime 2}\right) \\
& \left.-\frac{i \epsilon}{2} \xi_{i}\left(Q_{i}+Q_{i}^{\prime}\right)-\frac{\epsilon^{2}}{4 \alpha} \sum_{j=0}^{N-1} G_{i j} \xi_{i} \xi_{j}\right\}= \\
& =\int d Q_{0} \int d Q_{0}^{\prime}\left\langle Q_{0}, t_{0}\left|\hat{\rho}_{0}\right| Q^{\prime} t_{0}\right\rangle \int_{Q_{0}}^{Q_{F}} \mathcal{D} Q \int_{Q_{0}^{\prime}}^{Q_{F}^{\prime}} \mathcal{D} Q^{\prime} \\
& \exp \left\{\sum_{i, j=0}^{N} \frac{1}{\epsilon} K_{i j}\left[-\frac{\alpha}{4}\left(Q_{i}-Q_{i}^{\prime}\right)\left(Q_{j}-Q_{j}^{\prime}\right)+\frac{i}{2} Q_{i} Q_{j}-\frac{i}{2} Q_{i}^{\prime} Q_{j}^{\prime}\right]\right. \\
& \left.-\frac{i \epsilon}{2} \sum_{j=0}^{N-1} \xi_{j}\left(Q_{j}+Q_{j}^{\prime}\right)-\frac{\epsilon^{2}}{4 \alpha} \sum_{i, j=0}^{N-1} \xi_{i} G_{i j} \xi_{j}\right\},
\end{aligned}
$$

the limit $N \rightarrow \infty$ being obviously understood. Here we have set $\mathcal{D} Q=$ $\left(\frac{1}{2 \pi \epsilon}\right)^{\frac{N}{2}} \prod_{j=1}^{N-1} d Q_{j}, Q_{N}=Q_{F}, Q_{N}^{\prime}=Q_{F}^{\prime}$, we have denoted by $K_{i j}$ the 
$(N+1) \times(N+1)$ matrix

$$
K_{i j}=\left(\begin{array}{cccccc}
1-\epsilon^{2} \omega^{2} & -1 & 0 & \ldots & 0 & 0 \\
-1 & 2-\epsilon^{2} \omega^{2} & -1 & \ldots & 0 & 0 \\
0 & -1 & 2-\epsilon^{2} \omega^{2} & \ldots & 0 & 0 \\
\ldots & \ldots & \ldots & \ldots & \ldots & \ldots \\
0 & 0 & 0 & \ldots & 2-\epsilon^{2} \omega^{2} & -1 \\
0 & 0 & 0 & \ldots & -1 & 1
\end{array}\right)
$$

and we have assumed $G_{i j}=\epsilon\left(\bar{K}^{-1}\right)_{i j}, \bar{K}_{i j}$ being the $N \times N$ matrix obtained by suppressing the last row and column in $K_{i j}$.

Note that, while $\left.\operatorname{det} K_{i j}\right|_{\epsilon=0}=0$, we have $\left.\operatorname{det} \bar{K}_{i j}\right|_{\epsilon=0}=1$ and the matrix $\left.\bar{K}_{i j}\right|_{\epsilon=0}$, which corresponds to kinetic energy alone, it is non singular and positive. It can be expected that the matrix $\bar{K}_{i j}$ itself is also positive for $\epsilon=T / N=\left(t_{F}-t_{0}\right) / N$ if $T<T_{P}$, consistently with the fact that in such a situation the smallest non vanishing frequencies $\left|k_{ \pm 1}\right|=2 \pi / T$ in the Fourier expansion of $K(t-t)$ are larger than $\omega .{ }^{3}$ For the more interesting case of $T \gg T_{P}$, the matrix $\bar{K}_{i j}$ should develop negative eigenvalues. Consequently the integral in (69) is not absolutely convergent in such a general case. Due to the prevalence of the terms in $1 / \epsilon$ in the exponential, however, it remain convergent if performed step by step. ${ }^{4}$

Now, let us assume for the moment $T<T_{P}$ and replace (69) in the analog of (23). Then, even the matrix $G_{i j}$ is positive and the integral over $\xi_{0}, \xi_{1}, \ldots \xi_{N-1}$ is convergent and can be performed explicitly, being of Gaussian type. The expression for $\mathbf{f}\left(t_{F}, t_{0} ;[q(t)]\right)$ that we obtain in this way can be written in a particularly significant form if we set

$$
q_{N} \equiv q_{F}=\frac{Q_{F}+Q_{F}^{\prime}}{2} .
$$

We have

$$
\begin{aligned}
& \left\langle Q_{F}, t_{F}\left|\mathbf{f}\left(t_{F}, t_{0} ;[q(t)]\right) \hat{\rho}_{0}\right| Q_{F}^{\prime}, t_{F}\right\rangle=\left(\frac{2 \alpha}{\epsilon^{2}}\right)^{\frac{N}{2}} \int d Q_{0} d Q_{0}^{\prime} \\
& \int_{Q_{0}}^{Q_{F}} \mathcal{D} Q \exp \sum_{j=0}^{N-1}\left\{-\frac{\alpha}{2}\left[\frac{1}{\epsilon}\left(\left(q_{i+1}-Q_{i+1}\right)-\left(q_{i}-Q_{i}\right)\right)^{2}\right.\right. \\
& \left.\left.-\omega^{2} \epsilon\left(q_{i}-Q_{i}\right)^{2}\right]+\frac{i}{2}\left[\frac{1}{\epsilon}\left(Q_{i+1}-Q_{i}\right)^{2}-\omega^{2} Q_{i}^{2}\right]\right\}\left\langle Q_{0}\left|\hat{\rho}_{0}\right| Q_{0}^{\prime}\right\rangle
\end{aligned}
$$

\footnotetext{
${ }^{3}$ A direct proof that $\bar{K}_{i j}$ is positive can be done under a somewhat more restrictive condition than $T<T_{p}$. Note that, in order the matrix to be positive, it is necessary that $\epsilon^{2} \omega^{2}$ is smaller than $\lambda_{m}$, the minimum eigenvalue of $\left.\bar{K}_{i j}\right|_{\epsilon=0}$. Now such eigenvalues are given by the roots of a polynomial of the form $\left.\operatorname{det} \bar{K}_{i j}\right|_{\epsilon=0}-\lambda \delta_{i j}=A_{0}-A_{1} \lambda+A_{2} \lambda^{2}-$ $\ldots+(-\lambda)^{N}$, where the coefficients $A_{0}, A_{1}, \ldots$ are all positive and in, particular, $A_{0}=1$, $A_{1}=N(N+1) / 2$. Then we have $\lambda_{m}>A_{0} / A_{1}=2 / N(N+1)$ and $\epsilon^{2} \omega^{2} \equiv \frac{\omega^{2} T^{2}}{N^{2}}<\frac{2}{N(N+1)}$ if $T<\sqrt{2} / \omega$.

4 The situation is completely similar to that encountered in the calculation of the amplitude $\left\langle Q_{F}, t_{F} \mid Q_{0}, t_{0}\right\rangle$ for the harmonic oscillator, that can be performed exactly (see [21]) both for a real or for an imaginary mass $m=i \mu$ with $\mu>0$.
} 


$$
\begin{gathered}
\int_{Q_{0}^{\prime}}^{Q_{F}^{\prime}} \mathcal{D} Q^{\prime} \exp \sum_{i=0}^{N-1}\left\{-\frac{\alpha}{2}\left[\frac{1}{\epsilon}\left(\left(q_{i+1}-Q_{i+1}^{\prime}\right)-\left(q_{i}-Q_{i}^{\prime}\right)\right)^{2}-\right.\right. \\
\left.\left.-\omega^{2} \epsilon\left(q_{i}-Q_{i}^{\prime}\right)^{2}\right]+\frac{i}{2}\left[\frac{1}{\epsilon}\left(Q_{i+1}^{\prime}-Q_{j}^{\prime}\right)^{2}-\omega^{2} \epsilon{Q_{j}^{\prime}}^{2}\right]\right\}
\end{gathered}
$$

The positivity of $G_{i j}$ even implies

$$
\sum_{i, j=1}^{N-1} \xi_{i} G_{i j} \xi_{j}>0,
$$

for any $\left(\xi_{1}, \xi_{2}, \ldots \xi_{N-1}\right)$.

For $T>T_{P}$, we have no direct argument for the integral over $\xi_{0}, \xi_{1}, \ldots \xi_{N-1}$ to be at least conditionally convergent. However, we can assume so, on the basis of consistency arguments. In fact, note that eq. (72) makes sense even for $T>T_{P}$ and the Fourier transform $\int \mathcal{D}_{c} q \exp \left\{-i \sum_{j=0}^{N-1} \xi_{j} q_{j}\right\} \mathbf{f}\left(t_{F}, t_{0} ;[q]\right)$ converges again step by step, at least for $\xi_{0}, \xi_{1}, \ldots \xi_{N-1}$ satisfying eq. (73) (now reinterpreted as a condition equation), and reproduces eq. (69).

Concerning eq. (72), note that, if it were not for condition (71), it would be of the form (29). Even so, let us observe that for $Q_{F}=Q_{F}^{\prime}$ its right hand side is a positive quantity, if $\hat{\rho}_{0}$ is a positive operator (as we always assume). Then, the functional density of probability for the histories $q(t)$ of the macroscopic position,

$$
p\left(t_{F}, t_{0} ;[q(t)]\right)=\operatorname{Tr}\left\{\mathbf{f}\left(t_{F}, t_{0} ;[q(t)]\right) \hat{\rho}_{0}\right\},
$$

is positive and this is what we had to prove.

As usual, in the limit $N \rightarrow \infty$ eqs. (69) and (72) can be formally written in a continuous notation as

$$
\begin{aligned}
& \left\langle Q_{F}, t_{F}\left|\mathcal{G}\left(t_{F}, t_{0} ;[\xi]\right) \hat{\rho}_{0}\right| Q_{F}^{\prime}, t_{F}\right\rangle= \\
& =\int d Q_{0} \int d Q_{0}^{\prime}\left\langle Q_{0}, t_{0}\left|\hat{\rho}_{0}\right| Q^{\prime}, t_{0}\right\rangle \int_{Q_{0}}^{Q_{F}} \mathcal{D} Q \int_{Q_{0}^{\prime}}^{Q_{F}^{\prime}} \mathcal{D} Q^{\prime} \\
& \exp \int_{t_{0}}^{t_{F}} d t\left\{-\frac{\alpha}{4}\left[\left(\dot{Q}-\dot{Q}^{\prime}\right)^{2}-\omega^{2}\left(Q-Q^{\prime}\right)^{2}\right]+\right. \\
& +\frac{i}{2}\left(\dot{Q}^{2}-\omega^{2} Q^{2}\right)-\frac{i}{2}\left(\dot{Q}^{\prime 2}-\omega^{2} Q^{\prime 2}\right)- \\
& \left.\quad-\frac{i}{2} \xi\left(Q+Q^{\prime}\right)-\frac{1}{4 \alpha} \int_{t_{0}}^{t_{F}} d t^{\prime} \xi(t) G\left(t-t^{\prime}\right) \xi\left(t^{\prime}\right)\right\}
\end{aligned}
$$

and

$$
\begin{aligned}
& \left\langle Q_{F}, t_{F}\left|\mathbf{f}\left(t_{F}, t_{0} ;[q(t)]\right) \hat{\rho}_{0}\right| Q_{F}^{\prime}, t_{F}\right\rangle=\left(\frac{2 \alpha}{\epsilon^{2}}\right)^{\frac{N}{2}} \int d Q_{0} d Q_{0}^{\prime} \\
& \int_{Q_{0}}^{Q_{F}} \mathcal{D} Q \exp \int_{t_{0}}^{t_{F}} d t^{\prime}\left\{-\frac{\alpha}{2}\left[(\dot{q}-\dot{Q})^{2}-\omega^{2}(q-Q)^{2}\right]+\right.
\end{aligned}
$$




$$
\begin{gathered}
\left.+\frac{i}{2}\left(\dot{Q}^{2}-\omega^{2} Q^{2}\right)\right\}\left\langle Q_{0}\left|\hat{\rho}_{0}\right| Q_{0}^{\prime}\right\rangle \\
\int_{Q_{0}^{\prime}}^{Q_{F}^{\prime}} \mathcal{D} Q^{\prime} \exp \int_{t_{0}}^{t_{F}} d t^{\prime}\left\{-\frac{\alpha}{2}\left[\left(\dot{q}-\dot{Q}^{\prime}\right)^{2}-\omega^{2}\left(q_{j}-Q_{j}^{\prime}\right)^{2}\right]+\right. \\
\left.+\frac{i}{2}\left(\dot{Q}^{\prime 2}-\omega^{2} Q^{2}\right)\right\}
\end{gathered}
$$

with, obviously, the boundary condition $q\left(t_{F}\right)=\frac{Q_{F}+Q_{F}^{\prime}}{2}$.

At first sight the significance of eqs. (75) and $(76)$ could seem questionable, since the kernel $K\left(t-t^{\prime}\right)=-\left(\frac{d^{2}}{d t^{2}}+\omega^{2}\right) \delta\left(t-t^{\prime}\right)$ and its inverse $G\left(t-t^{\prime}\right)$ are not positive in the Hilbert space $L^{2}\left(t_{0}, t_{F}\right)$ if $T>2 \pi / \omega$. However, after our discussion about eqs. (69) and (72), what the circumstance really shows is that with the adopted definitions the Fourier components with $|k|>\omega$ of the functions of interest,

$$
Q(t)-Q_{c}(t), \quad Q^{\prime}(t)-Q_{c}^{\prime}(t), \quad \xi(t), \quad q(t)-\frac{\left.Q(t)+Q^{\prime}(t)\right)}{2},
$$

must dominate the integrals $\left(Q_{c}(t)\right.$ and $Q_{c}^{\prime}(t)$ denoting the solutions of the classical equation of motion which interpolates $Q_{0}$ and $Q_{F}$ and $Q_{0}^{\prime}$ and $Q_{F}^{\prime}$ respectively). ${ }^{5}$

To be able to proceed directly in the appealing continuous formalism, by using only general properties of the functional integral (like its translational invariance), we have simply to assume that with the appropriate definitions all the integrals can be made convergent and, for consistency, that only $\xi(t)$ are admissible that satisfies the condition

$$
\begin{array}{rl}
\int_{t_{0}}^{t_{F}} & d t \int_{t_{0}}^{t_{F}} d t^{\prime} \xi(t) G\left(t-t^{\prime}\right) \xi\left(t^{\prime}\right)= \\
& =\sum_{k=-\infty}^{\infty} \tilde{\xi}_{k}^{*} \frac{1}{k^{2}-\omega^{2}} \tilde{\xi}_{k} \sim \int_{\infty}^{\infty} d k \tilde{\xi}^{*}(k) \frac{1}{k^{2}-\omega^{2}} \tilde{\xi}(k)>0
\end{array}
$$

$\tilde{\xi}_{k}$ 's being the Fourier coefficients and $\tilde{\xi}(k) \sim \sqrt{\frac{2 \pi}{T}} \tilde{\xi}_{k}$ the Fourier transform (meaning again that the components with $|k|>\omega$ are dominating in $\xi(t)$ ).

Now, note that, with definition $(61), \mathcal{L}^{\prime}(t) \hat{Q}(t)$ and $\mathcal{L}^{\prime}(t) \hat{P}(t)$ vanish. Then, $\langle\hat{Q}(t)\rangle_{\mathrm{QM}}$ remains a solution of the classical equation as the analog in ordinary Quantum Mechanics and we can write

$$
\langle q(t)\rangle=\langle\hat{Q}(t)\rangle_{Q M}=C \cos (\omega t+\delta) .
$$

We have also (see (45))

$$
\left\langle\bar{q}_{h}(t)\right\rangle=-\left.i \frac{\partial}{\partial k} \operatorname{Tr}\left\{\mathcal{G}\left(t_{F}, t_{0} ;[k h]\right) \hat{\rho}_{0}\right\}\right|_{k=0}=\left\langle\hat{Q}_{h}(t)\right\rangle_{\mathrm{QM}}
$$

\footnotetext{
${ }^{5}$ Note that under the restriction of $Q(t)$ to the subspace with Fourier components with $|k|>\omega$ alone the classic action is actually minimal for $Q(t)=Q_{c}(t)$.
} 
and, for a support of $h(t)$ sufficiently small as in eq. (48),

$$
\begin{aligned}
\left\langle\left(\bar{q}_{h}-\left\langle\bar{q}_{h}\right\rangle\right)^{2}\right\rangle & =-\left.\frac{\partial^{2}}{\partial k^{2}} \operatorname{Tr}\left\{\mathcal{G}\left(t_{F}, t_{0} ;[k h]\right) \rho_{0}\right\}\right|_{k=0}-\left\langle q_{h}\right\rangle^{2}= \\
& =\frac{G_{h h}}{2 \alpha}+\left\langle\left(\bar{q}_{h}-\left\langle\hat{Q}_{h}\right\rangle\right)^{2}\right\rangle_{\mathrm{QM}} .
\end{aligned}
$$

where

$$
G_{h h}=\int d t \int d t^{\prime} h(t) G\left(t-t^{\prime}\right) h\left(t^{\prime}\right)=\int d k \tilde{h}^{*}(k) \frac{1}{k^{2}-\omega^{2}} \tilde{h}(k) .
$$

According to eq. (38), we can have also

$$
\begin{aligned}
& p\left(\bar{q}_{1}, h_{1} ; \bar{q}_{2}, h_{2} ; \ldots \bar{q}_{l}, h_{l}\right)=\int \mathcal{D} q \delta\left(\bar{q}_{1}-q_{h_{1}}\right) \ldots \delta\left(\bar{q}_{l}-q_{h_{l}}\right) p\left(t_{F}, t_{0} ;[q(t)]\right) \\
& =\frac{1}{(2 \pi)^{l}} \int d k_{1} d k_{2} \ldots d k_{l} e^{i\left(k_{1} \bar{q}_{1}+\ldots k_{l} \bar{q}_{l}\right)} \operatorname{Tr}\left\{\mathcal{G}\left(t_{F}, t_{0} ;\left[k_{1} h_{1}+\ldots k_{l} h_{l}\right]\right) \hat{\rho}_{0}\right\} \\
& =\int d Q_{F} \int d Q_{0} \cdot d Q_{0}^{\prime}\left\langle Q_{0}, t_{0}\left|\hat{\rho}_{0}\right| Q_{0}^{\prime}, t_{0}\right\rangle \int_{Q_{0}}^{Q_{F}} \mathcal{D} Q \int_{Q_{0}^{\prime}}^{Q_{F}} \mathcal{D} Q^{\prime} \exp \int_{t_{0}}^{t_{F}} d t \\
& \left\{\frac{-\alpha}{4}\left[\left(\dot{Q}-\dot{Q}^{\prime}\right)^{2}-\omega^{2}\left(Q-Q^{\prime}\right)^{2}\right]+\frac{i}{2}\left(\dot{Q}^{2}-\omega^{2} Q^{2}\right)-\frac{i}{2}\left(\dot{Q}^{\prime 2}-\omega^{2} Q^{\prime 2}\right)\right\} \\
& \left(\frac{\alpha}{\pi}\right)^{\frac{l}{2}} \frac{1}{\left.\left(\operatorname{det} G_{h_{r} h_{s}}\right)\right)^{1 / 2}} \exp \left\{-\alpha \sum_{r s}\left(\bar{q}_{r}-\frac{Q_{h_{r}}+Q_{h_{r}}^{\prime}}{2}\right)\right. \\
& \left.G_{h_{r} h_{s}}^{-1}\left(\bar{q}_{s}-\frac{Q_{h_{s}}+Q_{h_{s}}^{\prime}}{2}\right)\right\}
\end{aligned}
$$

with

$$
G_{h_{r} h_{s}}=\int d t \int d t^{\prime} h_{r}(t) G\left(t-t^{\prime}\right) h_{s}\left(t^{\prime}\right)
$$

For what concerns the choice of the weight functions, note that, according to condition (78), $h(t)$ and $h_{1}(t), \ldots h_{l}(t)$ must be such that $G_{h h}$ or the matrix $G_{h_{r} h_{s}}$ were positive. It is clear that eq. (46) does not longer provides correct choices. Admissible functions can be obtained e. g. by multiplying any one of the functions of eq. (46) for $\cos \bar{k} t$ with $\bar{k}$ sufficiently larger than $\omega$ (this has the effect of shifting the Fourier spectrum of $h(t)$ toward the values $\bar{k}$ and $-\bar{k})$. Alternatively, one could multiply directly the Fourier transform $\tilde{h}(k)$ for a step function of the type $\theta\left(k^{2}-\kappa^{2}\right)$ with an appropriate $\kappa$.

As an example let us set

$$
h(t)=\frac{1}{\tau \sqrt{\pi}} e^{-\frac{t^{2}}{\tau^{2}}} \cos \bar{k} t,
$$

which has the Fourier transform

$$
\tilde{h}(k)=\frac{1}{\sqrt{8 \pi}}\left(e^{-\frac{\tau^{2}}{4}(k-\bar{k})^{2}}+e^{-\frac{\tau^{2}}{4}(k+\bar{k})^{2}}\right) .
$$


Then, from (81) we have

$$
\begin{aligned}
\left\langle q_{h}(t)\right\rangle= & \int d t^{\prime} h\left(t-t^{\prime}\right)\left\langle\hat{Q}\left(t^{\prime}\right)\right\rangle_{Q M}= \\
& \left.=C e^{-\frac{\left(\omega^{2}+\bar{k}^{2}\right) \tau 2}{4}} \cosh \frac{\bar{k} \omega \tau^{2}}{2} \cos (\omega t+\delta)\right) .
\end{aligned}
$$

This reduces to $C \cos (\omega t+\delta)$ for $\tau \ll 1 / \bar{k}<1 / \omega$; on the contrary it becomes negligible for large $\tau$ or $\bar{k}$ (obviously the time average damps the high frequencies). For the intrinsic classic fluctuation, a rough estimate gives

$$
G_{h h} \sim \frac{1}{\tau \sqrt{8 \pi}} \frac{1+e^{-\frac{\bar{k}^{2} \tau^{2}}{2}}}{\bar{k}^{2}-\omega^{2}}<\frac{1}{\tau \sqrt{2 \pi}} \frac{1}{\bar{k}^{2}-\omega^{2}} .
$$

Completely similar results could be obtained, e. g. for $\tilde{h}(k)=\frac{1}{\sqrt{2 \pi}} e^{-\tau^{2} k^{2} / 4} \theta\left(k^{2}-\right.$ $\kappa^{2}$ ) or for any other admissible weight functions.

Finally note that, if in $(83,84) h_{r}(t)$ is identified with $h\left(t-t_{r}\right)$ with $h(t)$ given by (85) and $t_{r}-t_{s}$ is large with respect to $\tau$, the element $G_{h_{r} h_{s}}$ would become negligible. So if the sets $M$ and $N$ in eq. (7) are defined by weight function of this type, they would be uncorrelated and the equation would be valid, as anticipated, in the same approximation.

In the case of the free scalar field we assume similarly

$$
\mathcal{K}(x, j) \hat{\rho}=\mathcal{L}(x) \hat{\rho}-\frac{i}{2} j(x)\{\hat{\varphi}(x), \hat{\rho}\}-\frac{1}{2 \alpha} \int_{\sigma_{0}}^{\sigma} d^{4} x^{\prime} G_{m}\left(x-x^{\prime}\right) j(x) j\left(x^{\prime}\right),
$$

where

$$
G_{m}\left(x-x^{\prime}\right)=\frac{1}{(2 \pi)^{4}} \int d^{4} k \frac{1}{k^{2}-m^{2}} e^{-i k_{\mu}\left(x-x^{\prime}\right)^{\mu}}
$$

is the solution of the equation

$$
\left(\square-m^{2}\right) G_{m}\left(x-x^{\prime}\right)=\delta^{4}\left(x-x^{\prime}\right)
$$

and, therefore, the inverse of the differential operator $\left(\square-m^{2}\right)$ under appropriate restrictions.

Then, it can be checked that the compatibility condition (51) is still satisfied and we can proceed in a complete analogy on the oscillator case, with only obvious modifications, by using the standard field functional integration.

Directly in continuous notations, we have for the CFO

$$
\begin{aligned}
& \left\langle\varphi_{F}, \sigma_{F}\left|\mathcal{G}\left(\sigma_{F}, \sigma_{0} ;[j]\right) \hat{\rho}_{0}\right| \varphi_{F}^{\prime}, \sigma_{F}\right\rangle= \\
& =\int \mathcal{D}_{\sigma_{0}} \varphi_{0} \int \mathcal{D}_{\sigma_{0}} \varphi^{\prime}\left\langle\varphi_{0}, \sigma_{0}\left|\hat{\rho}_{0}\right| \varphi_{0}^{\prime}, \sigma_{0}\right\rangle \int_{\varphi_{0}}^{\varphi_{F}} \mathcal{D} \varphi \int_{\varphi_{0}^{\prime}}^{\varphi_{F}^{\prime}} \mathcal{D} \varphi^{\prime} \\
& \exp \int_{\sigma_{0}}^{\sigma_{F}} d^{4} x\left\{-\frac{\alpha}{4}\left[\partial_{\mu}\left(\varphi-\varphi^{\prime}\right) \partial^{\mu}\left(\varphi-\varphi^{\prime}\right)-m^{2}\left(\varphi-\varphi^{\prime}\right)^{2}\right]\right. \\
& -\frac{i}{2} j\left(\varphi+\varphi^{\prime}\right)-\frac{1}{4 \alpha} \int_{\sigma_{0}}^{\sigma_{F}} d^{4} x^{\prime} j(x) G_{m}\left(x-x^{\prime}\right) j\left(x^{\prime}\right)+ \\
& \left.+\frac{i}{2}\left(\partial_{\mu} \varphi \partial^{\mu} \varphi-m^{2} \varphi^{2}\right)-\frac{i}{2}\left(\partial_{\mu} \varphi^{\prime} \partial^{\mu} \varphi^{\prime}-m^{2} \varphi^{\prime 2}\right)\right\}
\end{aligned}
$$


and now, what dominate are the Fourier components of $\varphi(x), \varphi^{\prime}(x)$ and $j(x)$ with $k^{2} \equiv k_{0}^{2}-\mathbf{k}^{2}>m^{2}$. Furthermore we have to restrict the external source $j(x)$ by the condition

$$
\int d^{4} x d^{4} x^{\prime} j(x) G_{m}\left(x-x^{\prime}\right) j\left(x^{\prime}\right)>0 .
$$

As above, we can express $\mathbf{f}\left(\sigma_{F}, \sigma_{0} ;[\phi(x)]\right)$ as a functional of the classic field $\phi(x)$

$$
\begin{gathered}
\left\langle\varphi_{F}, \sigma_{F}\left|\mathbf{f}\left(\sigma_{F}, \sigma_{0} ;[\phi(x)]\right) \hat{\rho}\right| \varphi_{F}^{\prime}, \sigma_{F}\right\rangle=C_{\alpha} \int \mathcal{D}_{\sigma_{0}} \varphi_{0} \int \mathcal{D}_{\sigma_{0}} \varphi^{\prime} \\
\int_{\varphi_{0}}^{\varphi_{F}} \mathcal{D} \varphi \exp \left\{-\frac{\alpha}{2} \int d^{4} x\left[\partial_{\mu}(\phi-\varphi) \partial^{\mu}(\phi-\varphi)\right.\right. \\
\left.\left.\quad-m^{2}(\phi-\varphi)^{2}\right]+\frac{i}{2}\left(\partial_{\mu} \varphi \partial^{\mu} \varphi-m^{2} \varphi^{2}\right)\right\}\left\langle\varphi_{0}, \sigma_{0}\left|\hat{\rho}_{0}\right| \varphi_{0}^{\prime}, \sigma_{0}\right\rangle \\
\int_{\varphi_{0}^{\prime}}^{\varphi_{F}^{\prime}} \mathcal{D} \varphi^{\prime} \exp \left\{-\frac{\alpha}{2} \int d^{4} x\left[\partial_{\mu}(\phi-\varphi) \partial^{\mu}(\phi-\hat{\varphi})\right.\right. \\
\left.\left.-m^{2}(\phi-\hat{\varphi})^{2}\right]-\frac{i}{2}\left(\partial_{\mu} \varphi^{\prime} \partial^{\mu} \varphi^{\prime}-m^{2} \varphi^{\prime 2}\right)\right\} .
\end{gathered}
$$

and we have

$$
\langle\phi(x)\rangle=\langle\hat{\varphi}(x)\rangle_{\mathrm{QM}}
$$

and also

$$
\left\langle\left(\phi_{h}(x)-\left\langle\phi_{h}(x)\right\rangle\right)^{2}\right\rangle=\frac{1}{2 \alpha} G_{h h}+\left\langle\left(\hat{\varphi}_{h}(x)-\left\langle\hat{\varphi}_{h}(x)\right\rangle\right)^{2}\right\rangle_{\mathrm{QM}},
$$

with

$$
G_{h h}=\int d^{4} x d^{4} x^{\prime} h(x) G_{m}\left(x-x^{\prime}\right) h\left(x^{\prime}\right) .
$$

Again $G_{h h}$ has to be positive and permitted choices for $h(x)$ would be e.g.

$h(x)=\frac{1}{\pi^{2}} \frac{1}{\tau a^{3}} e^{-\left(\frac{t^{2}}{\tau^{2}}+\frac{\mathbf{x}^{2}}{a^{2}}\right)} \cos \bar{k} t \quad$ or $\quad \tilde{h}(k)=\frac{1}{(2 \pi)^{2}} e^{-\frac{1}{4}\left(\tau^{2} k_{0}^{2}+a^{2} \mathbf{k}^{2}\right)} \theta\left(k^{2}-\mu^{2}\right)$,

with $\bar{k}^{2}$ sufficiently larger than $m^{2}+1 / a^{2}$ and an appropriate $\mu$, possibly somewhat smaller than $m$. With both choices $G_{h h} \sim \frac{1}{(2 \pi)^{2}} \frac{1}{\tau a^{3}} \frac{1}{k^{2}-m^{2}}$, where $\bar{k}$ stays for some value larger than $m$ in the second case.

\section{Spinor Electrodynamics}

Finally we want introduce a classical e. $\mathrm{m}$. field in the context of spinor electrodynamics. As we mentioned, we do not consider any classical variable relative to Dirac fields and any observation on the system is supposed to 
be expressed in terms of modifications on such a classical e. m. field (see appendix).

In the case, the form of $\mathcal{L}(x)$ is essentially determined by Lorentz and gauge invariance requirements. We set

$$
\begin{aligned}
\mathcal{L}(x) \hat{\rho} & =\frac{\gamma}{8}\left[\hat{F}_{\mu \nu}(x),\left[\hat{F}^{\mu \nu}(x), \hat{\rho}\right]\right]= \\
& =-\frac{\gamma}{4}\left(\left[\hat{E}^{i}(x),\left[\hat{E}^{i}(x), \hat{\rho}\right]\right]-\left[\hat{B}^{i}(x),\left[\hat{B}^{i}(x), \hat{\rho}\right]\right]\right),
\end{aligned}
$$

where

$$
\begin{array}{r}
F_{\mu \nu}(x)=\partial_{\mu} A_{\nu}(x)-\partial_{\nu} A_{\mu}(x) \\
E^{i}=F_{0 i} \quad B^{i}=\frac{1}{2} \epsilon_{i j l} F_{j l}
\end{array}
$$

and $\gamma$ is again a dimensionless constant. Notice that for the latter we have used a specific notation to stress the fact that in a realistic interpretation of the model it should be understood as a new fundamental constant of nature.

Eq. (99) turns out to be consistent with local energy, momentum and charge conservation; however, it corresponds again to coefficients $\alpha_{j}$ of both signs in (2) or (21). We are in a situation similar to the cases considered in sec. 4 and we can proceed in a very similar way.

We set

$$
\begin{aligned}
& \mathcal{K}\left(x, j_{\rho}(x)\right) \hat{\rho}=\mathcal{L}(x) \hat{\rho}+ \\
&+\frac{i}{2} j_{\mu}(x)\left\{\hat{A}^{\mu}(x), \hat{\rho}\right\}+\frac{1}{2 \gamma} \int_{\sigma_{0}}^{\sigma} d^{4} x^{\prime} j_{\mu}(x) G^{\mu \nu}\left(x-x^{\prime}\right) j_{\nu}\left(x^{\prime}\right) \hat{\rho}
\end{aligned}
$$

where $G^{\mu \nu}\left(x-x^{\prime}\right)$ is the Green function relative to the differential operator acting on the potential $A^{\mu}(x)$ in the equation of motion. This obviously depends on the gauge we use.

If we define $G_{0}\left(x-x^{\prime}\right)$ as in (90) with $m=0$, in the Coulomb Gauge we have

$$
\begin{aligned}
G^{00}\left(x-x^{\prime}\right) & =-\frac{1}{2 \pi} \frac{1}{\left|\mathbf{x}-\mathbf{x}^{\prime}\right|} \delta\left(t-t^{\prime}\right), \quad G^{0 i}\left(x-x^{\prime}\right)=G^{i 0}\left(x-x^{\prime}\right)=0 \\
G^{i j}\left(x-x^{\prime}\right) & =-\left(\delta_{i j}-\partial_{i} \frac{1}{\nabla^{2}} \partial_{j}\right) G_{0}\left(x-x^{\prime}\right)= \\
= & -\frac{1}{(\pi)^{4}} \int d^{4} k\left(\delta_{i j}-\frac{k_{h} k_{k}}{\mathbf{k}^{2}}\right) \frac{1}{k^{2}} e^{-i k\left(x-x^{\prime}\right)}
\end{aligned}
$$

and in a generic Lorentz gauge

$$
G_{\lambda}^{\mu \nu}\left(x-x^{\prime}\right)=\frac{1}{(4 \pi)^{4}} \int d^{4} k\left(g^{\mu \nu}-(1-\lambda) \mathrm{P} \frac{k_{\mu} k_{\nu}}{k^{2}}\right) \frac{1}{k^{2}} e^{-i k\left(x-x^{\prime}\right)},
$$

with the $\lambda$ specifying the specific choice. 
For consistency the classical source must be assumed to satisfy the continuity equation

$$
\partial_{\mu} j^{\mu}(x)=0
$$

and, in analogy on the cases of the harmonic oscillator and the scalar field, we can avoid an explicit reference to the complicate lattice formulation if we introduce the further restriction

$$
\int d^{4} x d^{4} x^{\prime} j_{\mu}(x) G^{\mu \nu}\left(x-x^{\prime}\right) j_{\nu}\left(x^{\prime}\right)=\int d^{4} \tilde{j}_{\mu}^{*}(k) \tilde{G}^{\mu \nu}(k) \tilde{j}_{\nu}(k)<0,
$$

the sign depending obviously on the Minkowskian metric.

We shall show that, under such assumptions, we can construct an operational density $\mathbf{f}\left(t_{F}, t_{0} ;\left[a^{\mu}\right]\right)$ in analogy on the preceding section $\left(a^{\mu}(x)\right.$ being the classical tetra-potential to which the classical e. $\mathrm{m}$. field is related by $\left.f^{\mu \nu}(x)=\partial^{\mu} a^{\nu}(x)-\partial^{\nu} a^{\mu}(x)\right)$ and from this we can derive a positive probability distribution on the space of the histories of the classical field.

The Lagrangian density of the system can be written as

$$
\begin{gathered}
L(x)=-\frac{1}{4} F_{\mu \nu} F^{\mu \nu}-\frac{i}{2}\left(\bar{\psi} \gamma^{\rho} \partial_{\rho} \psi-\partial_{\rho} \bar{\psi} \gamma^{\rho} \psi\right)- \\
-m \bar{\psi} \psi-e A^{\mu} \bar{\psi} \gamma^{\mu} \psi .
\end{gathered}
$$

Any number of Dirac fields could be included, however for simplicity we shall write explicitly only one.

Correspondingly for the energy momentum tensor we have

$$
\begin{aligned}
& T^{\mu \nu}(x)=T_{\mathrm{em}}^{\mu \nu}(x)+T_{\mathrm{D}}^{\mu \nu}(x)+T_{\mathrm{int}}^{\mu \nu}(x)= \\
& \quad=F^{\mu \rho} F_{\rho}^{\nu}+\frac{1}{4} g^{\mu \nu} F_{\rho \sigma} F^{\rho \sigma}+\frac{i}{2}\left(\bar{\psi} \gamma^{\mu} \partial^{\nu} \psi-\partial^{\nu} \bar{\psi} \gamma^{\mu} \psi\right)- \\
& \quad-g^{\mu \nu}\left[\frac{i}{2}\left(\bar{\psi} \gamma^{\rho} \partial_{\rho} \psi-\partial_{\rho} \bar{\psi} \gamma^{\rho} \psi\right)-m \bar{\psi} \psi\right]+g^{\mu \nu} e \bar{\psi} \gamma^{\rho} \psi A_{\rho}
\end{aligned}
$$

In particular we can write

$$
T_{\mathrm{em}}^{00}(x)=\frac{1}{2}\left(\mathbf{E}^{2}+\hat{\mathbf{B}}^{2}\right), \quad T_{\mathrm{em}}^{0 i}(x)=(\mathbf{E} \times \hat{\mathbf{B}})_{i} .
$$

Let us first work in Coulomb gauge, where the independent variables are in evidence.

We have two constraint equations

$$
\nabla \cdot \mathbf{A}(x)=0, \quad \nabla \cdot \mathbf{E}(x)=e \bar{\psi} \gamma^{0} \psi
$$

and the electric field can be split in a transversal and in a longitudinal part, $\mathbf{E}(x)=\mathbf{E}_{\mathrm{T}}(x)+\mathbf{E}_{\mathrm{L}}(x)$ with

$$
\begin{aligned}
\mathbf{E}_{\mathrm{T}}(x) & =-\frac{\partial \mathbf{A}(x)}{\partial t}, \quad \mathbf{E}_{\mathrm{L}}(x)=-\nabla A^{0}(x) \\
A^{0}(t, \mathbf{x}) & =-\frac{e}{4 \pi} \int d^{3} \mathbf{y} \frac{1}{|\mathbf{x}-\mathbf{y}|} \bar{\psi}(t, \mathbf{y}) \gamma^{0} \psi(t, \mathbf{y}) .
\end{aligned}
$$


Then, the independent variables are $\mathbf{A}(x)$, which is itself transversal, the transversal e. $\mathrm{m}$. field $\mathbf{E}_{\mathrm{T}}(x)$ and the Dirac field $\psi(x)$. The non zero basic commutation rules among such variables are

$$
\begin{gathered}
{\left[\hat{E}^{i}(t, \mathbf{x}), \hat{A}^{j}\left(t, \mathbf{x}^{\prime}\right)\right]=i\left(\delta_{i j}-\partial_{i} \frac{1}{\nabla^{2}} \partial_{j}\right) \delta^{3}\left(\mathbf{x}-\mathbf{x}^{\prime}\right),} \\
\left\{\hat{\psi}_{\alpha}(t, \mathbf{x}), \overline{\hat{\psi}}_{\beta}\left(t, \mathbf{x}^{\prime}\right)\right\}=\gamma_{\alpha \beta}^{0} \delta^{3}\left(\mathbf{x}-\mathbf{x}^{\prime}\right)
\end{gathered}
$$

From these the commutation relations for the auxiliary variables follow,

$$
\begin{aligned}
& {\left[\hat{A}^{0}(t, \mathbf{x}), \hat{A}^{i}\left(t, \mathbf{x}^{\prime}\right)\right]=0, \quad\left[\hat{A}^{0}(t, \mathbf{x}), \hat{E}_{\mathrm{T}}^{i}\left(t, \mathbf{x}^{\prime}\right)\right]=0,} \\
& {\left[\hat{E}_{L}^{i}(t, \mathbf{x}), \overline{\hat{\psi}}_{\alpha}\left(t, \mathbf{x}^{\prime}\right) \hat{\psi}_{\beta}\left(t, \mathbf{x}^{\prime}\right)\right]=0,}
\end{aligned}
$$

and for the total e. m. field

$$
\begin{aligned}
& {\left[\hat{E}^{i}(t, \mathbf{x}), \hat{E}^{j}\left(t, \mathbf{x}^{\prime}\right)\right]=0, \quad\left[\hat{B}^{i}(t, \mathbf{x}), \hat{B}^{j}\left(t, \mathbf{x}^{\prime}\right]=0\right.} \\
& {\left[\hat{E}^{i}(t, \mathbf{x}), \hat{B}^{j}\left(t, \mathbf{x}^{\prime}\right)\right]=-i \epsilon_{i j l} \partial_{l} \delta^{3}\left(\mathbf{x}-\mathbf{x}^{\prime}\right) .}
\end{aligned}
$$

The latter are obviously independent of the gauge.

From eq. (114) the consistency relation

$$
\left[\mathcal{K}\left(x, j_{\rho}(x)\right), \mathcal{K}\left(x^{\prime}, j_{\rho}\left(x^{\prime}\right)\right)\right]=0
$$

follow immediately on any space-like surface and we have also

$$
\mathcal{L}^{\prime}\left(x^{\prime}\right) \hat{T}^{0 \nu}(x)=0 .
$$

Consequently the local energy momentum conservation remains valid in the form

$$
\partial_{\mu}\left\langle\hat{T}^{\mu \nu}(x)\right\rangle_{Q M}=0
$$

and similar conservation equations can be written for the electric charge, the barionic number, etc.

Finally, according to the methods for path integral in gauge field theories the CFO can be written in the form

$$
\begin{aligned}
& \left\langle\mathbf{A}_{F}, \zeta_{F}, \sigma_{F}\left|\mathcal{G}\left(\sigma_{F}, \sigma_{0} ;\left[j_{\rho}\right]\right)\right| \mathbf{A}_{F}^{\prime}, \zeta_{F}^{\prime}, \sigma_{F}\right\rangle= \\
& =\sum_{\zeta_{0} \zeta_{0}^{\prime}} \int \mathcal{D}_{\sigma_{0}} \mathbf{A}_{0} \mathcal{D}_{\sigma_{0}} \mathbf{A}_{0}^{\prime}\left\langle\mathbf{A}_{0}, \zeta_{0}, \sigma_{0}\left|\hat{\rho}_{0}\right| \mathbf{A}_{0}^{\prime}, \zeta_{0}^{\prime}, \sigma_{0}\right\rangle \\
& \quad \int_{\mathbf{A}_{0}}^{\mathbf{A}_{F}} \mathcal{D} \mathbf{A} \delta[\nabla \cdot \mathbf{A}] \mathcal{D} \bar{\psi} \mathcal{D} \psi \int_{\mathbf{A}_{0}^{\prime}}^{\mathbf{A}_{F}^{\prime}} \mathcal{D} \mathbf{A}^{\prime} \delta\left[\nabla \cdot \mathbf{A}^{\prime}\right] \mathcal{D} \bar{\psi}^{\prime} \mathcal{D} \psi^{\prime} \\
& \exp \int_{\sigma_{0}}^{\sigma_{F}} d^{4} x\left\{\frac{\gamma}{16}\left(F_{\mu \nu}-F_{\mu \nu}^{\prime}\right)\left(F^{\mu \nu}-F^{\prime \mu \nu}\right)\right)+\frac{i}{4}\left(F_{\mu \nu} F^{\mu \nu}-F_{\mu \nu}^{\prime} F^{\prime \mu \nu}\right)- \\
& -\frac{i}{2} j_{\mu}\left(A^{\mu}+A^{\prime \mu}\right)+\frac{1}{2 \gamma} \int_{\sigma_{0}}^{\sigma_{F}} d^{4} x^{\prime} j_{\mu}(x) G^{\mu \nu}\left(x-x^{\prime}\right) j_{\nu}\left(x^{\prime}\right)+ \\
& \left.\quad+i\left[L(A, \bar{\psi}, \psi)-L\left(A^{\prime}, \bar{\psi}^{\prime}, \psi^{\prime}\right)\right]\right\},
\end{aligned}
$$


where $\zeta_{0}, \zeta_{0}^{\prime}, \zeta_{F}, \zeta_{F}^{\prime}$ specify initial and final states of the spinor field. Furthermore, consistently with (104), we can assume

$$
j_{0}(x)=0, \quad \nabla \cdot \mathbf{j}(x)=0
$$

and then (102) becomes

$$
\int d^{4} k \tilde{j}^{*}(k)\left(\delta_{i j}-\frac{k_{i} k_{j}}{\mathbf{k}^{2}}\right) j_{j}(k) \frac{1}{k^{2}}=\int d^{4} k|\mathbf{j}(k)|^{2} \frac{1}{k^{2}}>0,
$$

which essentially implies that the time-like $k$ 's prevail on space-like ones.

Note that in a generic Lorentz gauge eq. (118) can be rewritten as

$$
\begin{gathered}
\left\langle\mathbf{A}_{F}, \zeta_{F}, \sigma_{F}\left|\mathcal{G}\left(\sigma_{F}, \sigma_{0} ;\left[j_{\rho}\right]\right)\right| \mathbf{A}_{F}^{\prime}, \zeta_{F}^{\prime}, \sigma_{F}\right\rangle= \\
=\sum_{\zeta_{0} \zeta_{0}^{\prime}} \int \mathcal{D}_{\sigma_{0}} \mathbf{A}_{0} \mathcal{D}_{\sigma_{0}} \mathbf{A}_{0}^{\prime}\left\langle\mathbf{A}_{0}, \zeta_{0}, \sigma_{0}\left|\hat{\rho}_{0}\right| \mathbf{A}_{0}^{\prime}, \zeta_{0}^{\prime}, \sigma_{0}\right\rangle \\
\int_{\mathbf{A}_{0}}^{\mathbf{A}_{F}} \mathcal{D} A \mathcal{D} \bar{\psi} \mathcal{D} \psi \int_{\mathbf{A}_{0}^{\prime}}^{\mathbf{A}_{F}^{\prime}} \mathcal{D} A^{\prime} \mathcal{D} \bar{\psi}^{\prime} \mathcal{D} \psi^{\prime} \\
\exp \int_{\sigma_{0}}^{\sigma_{F}} d^{4} x\left\{\frac{\gamma}{8}\left(F_{\mu \nu}-F_{\mu \nu}^{\prime}\right)\left(F^{\mu \nu}-F^{\prime \mu \nu}\right)\right)- \\
-\frac{i}{2} j_{\mu}\left(A^{\mu}+A^{\prime \mu}\right)+\frac{1}{4 \gamma} \int_{\sigma_{0}}^{\sigma_{F}} d^{4} x^{\prime} j_{\mu}(x) G_{\lambda}^{\mu \nu}\left(x-x^{\prime}\right) j_{\nu}\left(x^{\prime}\right)+ \\
\left.\quad+i\left[L_{\mathrm{eff}}(A, \bar{\psi}, \psi)-L_{\mathrm{eff}}\left(A^{\prime}, \bar{\psi}^{\prime}, \psi^{\prime}\right)\right]\right\}
\end{gathered}
$$

where

$$
L_{\mathrm{eff}}(A, \bar{\psi}, \psi)=L(A, \bar{\psi}, \psi)-\frac{1}{2 \lambda}\left(\partial_{\mu} A^{\mu}\right),
$$

$G_{\lambda}^{\mu \nu}\left(x-x^{\prime}\right)$ is now defined by eq. (103) and for the classical source $j_{\mu}(x)$ we have to assume eqs. (104-105) in their original form.

From (118) or (121) we can obtain $\mathbf{f}\left(\sigma_{F}, \sigma_{0} ;\left[a^{\mu}(x)\right]\right)$, that we write directly in the operator form

$$
\begin{aligned}
& \left\langle\mathbf{A}_{F}, \zeta_{F}, \sigma_{F}\left|\mathbf{f}\left(\sigma_{F}, \sigma_{0} ;\left[a^{\mu}(x)\right]\right) \hat{\rho}\right| \mathbf{A}_{F}^{\prime}, \zeta_{F}^{\prime}, \sigma_{F}\right\rangle= \\
& =C_{\gamma}\left\langle\mathbf{A}_{F}, \zeta_{F}, \sigma_{F}\right| \exp \left\{\frac{\gamma}{4} \int d^{4} x\left[f_{\mu \nu}(x)-\hat{F}_{\mu \nu}(x)\right]\left[f^{\mu \nu}(x)-\hat{F}^{\mu \nu}(x)\right]\right\} \hat{\rho} \\
& \quad \exp \left\{\frac{\gamma}{4} \int d^{4} x\left[f_{\mu \nu}(x)-\hat{F}_{\mu \nu}(x)\right]\left[f^{\mu \nu}(x)-\hat{F}^{\mu \nu}(x)\right]\right\}\left|\mathbf{A}_{F}^{\prime}, \zeta_{F}^{\prime}, \sigma_{F}\right\rangle,
\end{aligned}
$$

where the condition $a^{\mu}(x)=\frac{1}{2}\left(A_{F}^{\mu}(x)+A_{F}^{\prime \mu}(x)\right)$ has to be understood on $\sigma_{F}$. Obviously this provides a positive density of probability $p\left(\sigma_{F}, \sigma_{0} ;\left[a^{\mu}\right]\right)$.

Finally let us consider the fluctuations of the classical e. m. field around its expectation value. We can write

$$
\left.(-i)^{2} \frac{\delta}{\delta j^{\mu}(x)} \frac{\delta}{\delta j^{\nu}\left(x^{\prime}\right)} \operatorname{Tr}\left[\mathcal{G}\left(\sigma_{F}, \sigma_{0} ;\left[j_{\rho}\right]\right) \hat{\rho}_{0}\right]\right|_{j=0}=
$$




$$
\begin{aligned}
& =\frac{1}{2 \gamma} G_{\mu \nu}\left(x-x^{\prime}\right)+\theta\left(t-t^{\prime}\right) \frac{1}{2} \operatorname{Tr}\left[\hat{A}_{\mu}(x) \mathcal{G}\left(\sigma, \sigma^{\prime}\right)\left\{\hat{A}_{\nu}\left(x^{\prime}\right), \mathcal{G}\left(\sigma^{\prime}, \sigma_{0}\right) \hat{\rho}_{0}\right\}\right]+ \\
& \quad+\theta\left(t^{\prime}-t\right) \frac{1}{2} \operatorname{Tr}\left[\hat{A}_{\nu}\left(x^{\prime}\right) \mathcal{G}\left(\sigma^{\prime}, \sigma\right)\left\{\hat{A}_{\mu}(x), \mathcal{G}\left(\sigma, \sigma_{0}\right) \hat{\rho}_{0}\right\}\right] \cong \\
& \quad \cong \frac{1}{2 \gamma} G_{\mu \nu}\left(x-x^{\prime}\right)+\left\langle A_{\mu}(x) A_{\nu}\left(x^{\prime}\right)\right\rangle_{\mathrm{QM}},
\end{aligned}
$$

if $t^{\prime}$ is sufficiently close to $t$, and

$$
\begin{aligned}
& \left\langle f_{\mu \nu}(x) f_{\rho \sigma}\left(x^{\prime}\right)\right\rangle=\frac{1}{2 \gamma}\left[\partial_{\mu} \partial_{\rho}^{\prime} G_{\nu \sigma}\left(x-x^{\prime}\right)-\partial_{\mu} \partial_{\sigma}^{\prime} G_{\nu \rho}\left(x-x^{\prime}\right)-\right. \\
& \left.\quad-\partial_{\nu} \partial_{\rho} G_{\mu \sigma}\left(x-x^{\prime}\right)+\partial_{\nu} \partial_{\sigma} G_{\mu \rho}\left(x-x^{\prime}\right)\left(x-x^{\prime}\right)\right]+ \\
& \quad+\left\langle\hat{F}_{\mu \nu}(x) \hat{F}_{\rho \sigma}\left(x^{\prime}\right)\right\rangle_{\mathrm{QM}} .
\end{aligned}
$$

Then, let us set $E_{\text {class }}^{i}(x)=f_{0 i}(x), B_{\text {class }}^{i}(x)=\frac{1}{2} \epsilon_{i j l} f_{j l}(x)$ and

$$
\mathbf{E}_{h}(x)=\int d^{4} x^{\prime} h\left(x-x^{\prime}\right) \mathbf{E}_{\text {class }}\left(x^{\prime}\right) \quad \mathbf{B}_{h}(x)=\int d^{4} x^{\prime} h\left(x-x^{\prime}\right) \mathbf{B}_{\text {class }}\left(x^{\prime}\right),
$$

with $h(x)$ as in (98) with $m=0$ and $\bar{k}^{2}>1 / a^{2}$. Obviously we have

$$
\left\langle\mathbf{E}_{\text {class }}(x)\right\rangle=\langle\hat{\mathbf{E}}(x)\rangle_{\mathrm{QM}}, \quad\left\langle\mathbf{B}_{\text {class }}(x)\right\rangle=\langle\hat{\mathbf{B}}(x)\rangle_{\mathrm{QM}}
$$

and

$$
\begin{aligned}
\left\langle\left( E_{h}^{i}\right.\right. & \left.\left.-\left\langle E_{h}^{i}\right\rangle\right)^{2}\right\rangle=\frac{1}{2 \gamma} \int d^{4} k \mathrm{P} \frac{k_{0}^{2}-k_{i}^{2}}{k^{2}}|\tilde{h}(k)|^{2}+\left\langle\left(\hat{E}_{h}^{i}-\left\langle\hat{E}_{h}^{i}\right\rangle\right)^{2}\right\rangle_{\mathrm{QM}} \sim \\
& \sim \frac{1}{2(2 \pi)^{2} \gamma \tau a^{3}}+\left\langle\left(\hat{E}_{h}^{i}-\left\langle\hat{E}_{h}^{i}\right\rangle\right)^{2}\right\rangle_{\mathrm{QM}},
\end{aligned}
$$

A similar equation can be derived for $B_{h}^{i}$.

\section{Conclusive consideration}

In conclusion we have shown on three different models that it is possible modify the formalism for the continuous monitoring of macroscopic quantities in Quantum Theory in such a way that the basic conservation laws and (when appropriate) covariance are preserved.

As we mentioned, the idea is that Quantum Theory should be modified by introducing certain basic macroscopic quantities, that are formally treated as continuously monitored but are actually thought as classical quantities or beables. These are supposed to have well determined values at each time and in terms of modifications of them any other observation should be expressed.

Obviously the only really significant of the models we have considered is Spinor Electrodynamics, in which the macroscopic electromagnetic field components are considered as classical. Even this, to be made realistic, should be extended at least to the particle physics Standard Model. In the minimal formulation of the latter, in which the Higgs is treated as elementary, this may be 
not a trivial task. The difficulty comes from the occurrence of terms quadratic in the e.m. potential in the boson sector of the theory that would violate eq. (116) and so again the conservation law (117); even if such violations should be very small, typically of the order $\gamma G_{F}$ ( $G_{F}$ being the Fermi constant). In any case some important properties of the model should remain valid in a more complete theory. Let discuss them very briefly.

First of all, note that it is implicitly built in the eqs. (7-9) that, when applied to a small number of particles, the formalism reproduces the usual quantum theory within a good approximation, if $\gamma$ is small. Two essential modifications occur:

1) only observables that can be expressed in terms of a modification of the above macroscopic field must be considered;

2) the usual unitary evolution has to be corrected by the action of the mapping $\mathcal{G}\left(t, t_{a}\right)$ on the initial density operator $\hat{\rho}\left(t_{a}\right)$.

In the context, small number of particles means a number compatible with a negligible macroscopic e. m. field in the volume occupied by the system.

A more detailed discussion is given in app. B. Here let us make only few comments.

Concerning point 1), simply note that practically all our particle detectors work in terms of e. m. effects, that by appropriate amplification reach the macroscopic scale. In last analysis, even in the spirit of von Neumann psycho-physical parallelism, the states of our brain related to our perceptions are expressed in terms of membrane potentials, action potentials, charge distributions and so on.

On point 2) let us observe that the size of the corrections to the time evolution is controlled by the value of the constant $\gamma$. As we mentioned, this should be intended as a new fundamental constant of nature, which in some way would establish the boundary between what is classical and what is quantum. Since ordinary quantum theory works well for few particles, it is clear that $\gamma$ has to be small. However in eq. (128) $\gamma$ occurs in the denominator of the expression of the variance of the field and in order the entire construction makes sense even the latter must be negligible at some typical macroscopic scale. So for some reasonable values of $\tau$ and $a$ in (128) and some appropriate $\mathbf{E}_{\text {typ }}$ or $\mathbf{B}_{\text {typ }}$ we must have

$$
\left\langle\left(\mathbf{E}_{h}-\left\langle\mathbf{E}_{h}\right\rangle\right)^{2}\right\rangle / \mathbf{E}_{\text {typ }}^{2} \ll 1, \quad\left\langle\left(\mathbf{B}_{h}-\left\langle\mathbf{B}_{h}\right\rangle\right)^{2}\right\rangle / \mathbf{B}_{\text {typ }}^{2} \ll 1 .
$$

This provide us the lower bound

$$
\gamma \gg 1.27 \times 10^{-2} /\left(\mathbf{E}_{\text {typ }}^{2} \tau a^{3}\right) .
$$

and a similar for $\mathbf{B}_{\text {typ }}$. To see what this means let us take $\mathbf{E}_{\text {typ }}^{2} \sim \mathbf{B}_{\text {typ }}^{2}$ as the value of the fields in equilibrium inside a cavity at ordinary temperature $T=300 \mathrm{~K}$ and e. g. $\tau=1 \mathrm{~ms}=3 \times 10^{7} \mathrm{~cm}, a=1 \mu \mathrm{m}=10^{-4} \mathrm{~cm}$. For the e. $\mathrm{m}$. energy density the Stefan-Boltzman law gives in natural units

$$
u(T)=7.56 \times 10^{-15} T^{4} \mathrm{erg} \mathrm{cm}^{-1}=2.39 \times 10^{2} T^{4} \mathrm{~cm}^{-4} .
$$


Then, setting

$$
\mathbf{E}_{\text {typ }}^{2} \sim \mathbf{B}_{\text {typ }}^{2} \sim u(300 \mathrm{~K})=1.94 \times 10^{12} \mathrm{~cm}^{-4}
$$

eq. (130) becomes

$$
\gamma \gg 2.2 \times 10^{-10}
$$

which seems consistent with a sufficiently small value of $\gamma$.

Note that the values we have assumed for $\tau$ and $a$ intend to refer to a macroscopic scale in the sense of our perception capability and not to the minimal scale at which the classical Maxwell equations can be applied, which is certainly much lower.

Finally, talking about experimental consequences of the theory, let us stress that an equation of the type (3) introduces an additional time inversion violation that could have astrophysical and cosmological consequences and should be investigated. Possibly it is just throughout such a kind of consequences that a theory of this type could be better tested and, in case, the constant $\gamma$ determined. At the moment we have not elaborated the subject. However, for what concerns the possibility of tests in the frame of optics or condensed matter physics, we may refer e. g. to the thorough discussion in [10], which concerns specifically the case of collapse models, but in part it applies even to the present theory.

\section{Acknowledgments}

Warm thanks are due to my friend L. Lanz for many interesting discussion and critical remarks.

\section{A A brief reference to Generalized Quantum Mechanics}

Let us recall that in GQM a set of compatible observables $A \equiv\left(A^{1}, A^{2}, \ldots A^{p}\right)$ is associated to a normalized effect or positive operator valued measure (p.o.m.) $\hat{F}_{A}(T)$ and the apparatus $S_{A}$ for observing them to an instrument or operation valued measure (o.v.m.) $\mathcal{F}_{S_{A}}(T), T$ being a Borel subset of the real space $\mathbb{R}^{p}$ of all possible values of $A$. That is, $\hat{F}_{A}(T)$ and $\mathcal{F}_{S_{A}}(T)$ are a positive operator on the Hilbert space $\mathbb{H}$ associated to the system and a positive mapping of the set of the trace class operators in itself, respectively, satisfying the relations

$$
\hat{F}\left(\cup_{j=1}^{n} T_{j}\right)=\sum_{j=1^{n}} \hat{F}\left(T_{j}\right) \quad \text { and } \quad \mathcal{F}\left(\cup_{j=1}^{n} T_{j}\right)=\sum_{j=1^{n}} \mathcal{F}\left(T_{j}\right),
$$

if $T_{i} \cap T_{j}=0$, and

$$
\hat{F}_{A}\left(\mathbb{R}^{p}\right)=\hat{I} \quad \operatorname{Tr}\left[\mathcal{F}_{S_{A}}\left(\mathbb{R}^{p}\right) \hat{X}\right]=\operatorname{Tr} \hat{X} .
$$

Further they must be related each other by the equation

$$
\hat{F}_{A}(T)=\mathcal{F}_{S_{A}}^{\prime}(T) \hat{I}
$$

where by $\mathcal{F}^{\prime}$ we denote the dual mapping of $\mathcal{F}$, defined by the equation

$$
\operatorname{Tr}[\hat{B} \mathcal{F} \hat{X}]=\operatorname{Tr}\left[\left(\mathcal{F}^{\prime} \hat{B}\right) \hat{X}\right],
$$


$\hat{X}$ being an arbitrary trace class operator and $\hat{B}$ an arbitrary bounded operator. So

$$
\operatorname{Tr}\left[\hat{F}_{A}(T) \hat{X}\right]=\operatorname{Tr}\left[\mathcal{F}_{S_{A}}(T) \hat{X}\right]
$$

As we told, we shall find convenient to work in Heisenberg picture. Then we have

$$
\begin{gathered}
\hat{F}_{A}(T, t)=e^{i H t} \hat{F}_{A}(T) e^{-i H t} \\
\mathcal{F}_{S_{A}}(T, t) \hat{X}=e^{i H t}\left[\mathcal{F}_{S_{A}}(T)\left(e^{-i H t} \hat{X} e^{i H t}\right)\right] e^{-i H t}
\end{gathered}
$$

and the probability of observing $A \in T$ at the time $t$ is

$$
P(A \in T, t)=\operatorname{Tr}\left[\hat{F}_{A}(T, t) \hat{\rho}\right]=\operatorname{Tr}\left[\mathcal{F}_{S_{A}}(T, t) \hat{\rho}\right],
$$

where $\hat{\rho}$ denotes the statistical operator representing the state of the system (a priori a mixture state.

The reduction of the state as consequence of having observed $A \in T$ at the time $t_{0}$ by the apparatus $S_{A}$ must be written as

$$
\hat{\rho} \rightarrow \mathcal{F}_{S_{A}}\left(T, t_{0}\right) \hat{\rho} / \operatorname{Tr}\left[\mathcal{F}_{S_{A}}\left(T, t_{0}\right) \hat{\rho}\right] .
$$

Notice

$$
\left\langle A^{j}\right\rangle=\operatorname{Tr}\left[\hat{A}^{j}(t) \hat{\rho}\right]
$$

with

$$
\hat{A}^{j}(t)=e^{i H t} \hat{A}^{j} e^{-i H t} \quad \text { and } \quad \hat{A}^{j}=\int_{\Re p} d \hat{F}(a) a^{j} .
$$

The operators $\hat{A}^{j}$ are Hermitian but generally they do not commute. Such a set of generalized compatible observables can be interpreted as corresponding to an approximate simultaneous measurement of possibly incompatible ordinary observables $\hat{A}_{1}, \hat{A}_{2}, \ldots$

Now let us assume that we make repeated independent observations on $A$ at subsequent times $t_{0}, t_{1}, \ldots t_{N}$. Combining eqs. (140) and (141) the Joint probability of observing a a sequence of results for $A$ can be written

$$
\begin{aligned}
& P\left(A \in T_{N}, t_{N} ; \ldots A \in T_{1}, t_{1} ; A \in T_{0}, t_{0}\right)= \\
& \quad=\operatorname{Tr}\left[\mathcal{F}_{S_{A}}\left(T_{N}, t_{N}\right) \ldots \mathcal{F}_{S_{A}}\left(T_{1}, t_{1}\right) \mathcal{F}_{S_{A}}\left(T_{0}, t_{0}\right) \hat{\rho}\right]
\end{aligned}
$$

Notice that

$$
\left.\mathcal{F}\left(T_{N}, t_{N} ; \ldots ; T_{1}, t_{1} ; T_{0}, t_{0}\right)=\mathcal{F}_{S_{A}}\left(T_{N}, t_{N}\right) \ldots \mathcal{F}_{S_{A}}\left(T_{1}, t_{1}\right) \mathcal{F}_{S_{A}}\left(T_{0}, t_{0}\right)\right)
$$

and

$$
\left.\hat{F}\left(T_{N}, t_{N} ; \ldots ; T_{1}, t_{1} ; T_{0}, t_{0}\right)=\mathcal{F}_{S_{A}}^{\prime}\left(T_{0}, t_{0}\right) \mathcal{F}_{S_{A}}^{\prime}\left(T_{1}, t_{1}\right) \ldots \mathcal{F}_{S_{A}}^{\prime}\left(T_{N}, t_{N}\right)\right) \hat{I}
$$

define an instrument and a p.o.m. on a real space with $p(N+1)$ dimensions $\Re^{p(N+1)}$.

Then

$$
\begin{aligned}
P\left(A \in T_{N}, t_{N} ; \ldots ; A \in T_{0}, t_{0}\right)= \\
\quad=\operatorname{Tr}\left[\mathcal{F}\left(T_{N}, t_{N} ; \ldots ; T_{0}, t_{0}\right) \hat{\rho}\right] \\
=\operatorname{Tr}\left[\hat{F}\left(T_{N}, t_{N} ; \ldots ; T_{0}, t_{0}\right) \hat{\rho}\right]
\end{aligned}
$$

So in GQM the observation of a sequence of results at certain successive times can be put on the same foot as the observation of $A$ at a single time. 


\section{B Recovery of ordinary Quantum Mechanics for a small system}

In the perspective of the paper, as we stressed, any observation on a system has to be expressed in terms of the modification that the system induces on the classical e. m. field.

Let us then consider, e. g., a system of a small number of particles, characterized by a certain set of invariants (a total electric charge, baryon number, lepton number, etc), to which we shall refer as the object. Let us assume that such particles interact freely among themselves during a certain interval of time $\left(t_{a}, t_{b}\right)$. We can admit any kind of rearrangement inside the system, exchange of energy and momentum, production or destruction of particles, but no interaction with the external environment during such interval of time.

We assume that at the time $t_{b}$ the system comes in contact with an apparatus, by which the specific type of final particles, their momenta, energies etc. can be detected. To be specific we may think of the apparatus as a set of counters, filling densely a certain region of the space possibly kept under the action of a magnetic field.

Both the object and the apparatus in their specific states must be thought as states of the same system of fields initially localized in different parts of the space. Such states can be expressed by appropriate composed creator operators applied to the vacuum state. Let us denote by $\left|u_{1}(t)\right\rangle,\left|u_{2}(t)\right\rangle, \ldots$ and $\left|U_{1}(t)\right\rangle,\left|U_{2}(t)\right\rangle, \ldots$ two orthogonal basis in the subspaces of the object system and of the apparatus at the time $t$ and write

$$
\left|u_{j}(t)\right\rangle=\hat{a}_{j}^{\dagger}(t)|0\rangle \quad\left|U_{r}(t)\right\rangle=\hat{A}_{r}^{\dagger}(t)|0\rangle
$$

$\hat{a}_{j}^{\dagger}(t)$ and $\hat{A}_{j}^{\dagger}(t)$ being ordinary Heisenberg picture operators which commute for $t<t_{b}$. Then let us assume the object described at the initial time $t_{a}$ by the statistical operator

$$
\begin{aligned}
\hat{\rho}^{\mathrm{O}}\left(t_{a}\right)= & \sum_{i j}\left|u_{i}\left(t_{a}\right)\right\rangle \rho_{i j}^{\mathrm{O}}\left(t_{a}\right)\left\langle u_{j}\left(t_{a}\right)\right|= \\
& =\sum_{i j} \hat{a}^{\dagger}\left(t_{a}\right)|0\rangle \rho_{i j}^{\mathrm{O}}\left(t_{a}\right)\langle 0| \hat{a}_{i}\left(t_{a}\right) .
\end{aligned}
$$

The assumption that the number of particles is small implies that the classical fields $\mathbf{E}_{\text {classic }}(t, \mathbf{x})$ and $\mathbf{B}_{\text {classic }}(t, \mathbf{x})$ remain negligible in the region occupied by the system until this does come in contact with the apparatus. Then, if $L_{0} \in \Sigma_{t_{a}}^{t_{b}}$ is the set of the histories of the field corresponding to such situation, the probability of occurrence of the complementary set must be null, $\mathcal{F}\left(L_{0}^{\prime} ; t_{b}, t_{a}\right) \hat{\rho}\left(t_{a}\right)=0$. So at the time $t_{b}$ we have

$$
\begin{aligned}
\hat{\rho}^{\mathrm{O}}\left(t_{b}\right)= & \mathcal{F}\left(L_{0} ; t_{b}, t_{a}\right) \hat{\rho}^{\mathrm{O}}\left(t_{a}\right)=\mathcal{G}\left(t_{b}, t_{a}\right) \hat{\rho}^{\mathrm{O}}\left(t_{a}\right)= \\
& =\sum_{i j} \hat{a}_{i}^{\dagger}\left(t_{b}\right)|0\rangle \rho_{i j}^{\mathrm{O}}\left(t_{b}\right)\langle 0| \hat{a}_{j}\left(t_{a}\right),
\end{aligned}
$$

where

$$
\rho_{i j}^{\mathrm{O}}\left(t_{b}\right)=\left\langle u_{i}\left(t_{b}\right)\left|\mathcal{G}\left(t_{b}, t_{a}\right)\left\{\sum_{k l} \hat{a}_{k}^{\dagger}\left(t_{a}\right)|0\rangle \rho_{k l}^{\mathrm{O}}\left(t_{a}\right)\langle 0| \hat{a}_{l}\left(t_{a}\right)\right\}\right| u_{j}\left(t_{b}\right)\right\rangle .
$$

Similarly let be

$$
\hat{\rho}^{\mathrm{A}}\left(t_{a}\right)=\sum_{r s} \hat{A}_{r}^{\dagger}\left(t_{a}\right)|0\rangle \rho_{r s}^{\mathrm{A}}\left(t_{a}\right)\langle 0| \hat{A}_{s}\left(t_{a}\right)
$$

the initial state of the apparatus. In this case we can assume that the counters remain in their charged states, corresponding to the classical e. m. field having certain specific stable values inside them, until any interaction with some external object occurs. Again this corresponds to the classical history of the e. m. field falling with certainty in an other set $M_{0} \in \Sigma_{t_{a}}^{t_{b}}$, being null the probability of occurrence of the complementary set. Then

$$
\hat{\rho}^{\mathrm{A}}\left(t_{b}\right)=\sum_{r s} \hat{A}_{r}^{\dagger}\left(t_{b}\right)|0\rangle \rho_{r s}^{\mathrm{A}}\left(t_{b}\right)\langle 0| \hat{A}_{s}\left(t_{b}\right)
$$


with

$$
\rho_{r s}^{\mathrm{A}}\left(t_{b}\right)=\left\langle U_{r}\left(t_{b}\right)\left|\mathcal{G}\left(t_{b}, t_{a}\right)\left\{\sum_{k l} \hat{A}_{k}^{\dagger}\left(t_{a}\right)|0\rangle \rho_{k l}^{\mathrm{A}}\left(t_{a}\right)\langle 0| \hat{A}_{l}\left(t_{a}\right)\right\}\right| U_{s}\left(t_{b}\right)\right\rangle .
$$

Finally, since we have assumed that the object and the apparatus do not come in contact before $t_{b}$, they must evolve independently during the interval $\left(t_{a}, t_{b}\right)$. Then, at the time $t_{b}$ we have for their compound state

$$
\begin{gathered}
\hat{\rho}^{\mathrm{T}}\left(t_{b}\right)=\sum_{i j} \sum_{r s} \hat{a}_{i}^{\dagger}\left(t_{b}\right) \hat{A}_{r}^{\dagger}\left(t_{b}\right)|0\rangle \rho_{i j}^{\mathrm{O}}\left(t_{b}\right) \rho_{r s}^{\mathrm{A}}\left(t_{b}\right)\langle 0| \hat{A}_{s}\left(t_{b}\right) \hat{a}_{j}\left(t_{b}\right)= \\
=\sum_{i j} \sum_{r s}\left|u_{i}, U_{r} ; t_{b}\right\rangle \rho_{i j}^{\mathrm{O}}\left(t_{b}\right) \rho_{r s}^{\mathrm{A}}\left(t_{b}\right)\left\langle u_{j}, U_{s} ; t_{b}\right| .
\end{gathered}
$$

In a subsequent time interval $\left(t_{b}, t_{c}\right)$, as consequence of the interaction with the particles of the object, some of the counter shall discharge and every specific pattern of discharged counters is interpreted as corresponding to certain specific particles with specific energies and momenta present in the system at time $t_{b}$. Then, if now we denote by $N \in \Sigma_{t_{b}}^{t_{c}}$ the set of classical e. m. world histories corresponding to the parameters specifying the particles types, energies, momenta etc. falling in a certain set $T$, we have

$$
\begin{aligned}
& p\left(T, t_{b}\right)=P\left(t_{c}, t_{b} ; N\right)= \\
& \operatorname{Tr}\left[\mathcal{F}\left(t_{c}, t_{b} ; N\right) \hat{\rho}^{\mathrm{T}}\left(t_{b}\right)\right]=\sum_{i j} F_{i j}\left(T, t_{b}\right) \rho_{j i}^{\mathrm{O}}\left(t_{b}\right),
\end{aligned}
$$

which is positive and where obviously

$$
\begin{aligned}
F_{i j}\left(T, t_{b}\right)= & \operatorname{Tr}\left[\mathcal { F } ( t _ { c } , t _ { b } ; N ) \left\{\sum_{r s}\left|u_{i}\left(t_{b}\right), U_{r}\left(t_{b}\right)\right\rangle\right.\right. \\
& \left.\left.\rho_{r s}^{\mathrm{A}}\left(t_{b}\right)\left\langle u_{j}\left(t_{b}\right), U_{s}\left(t_{b}\right)\right|\right\}\right] .
\end{aligned}
$$

To be more explicit, let us assume that the vectors $\left|u_{j}\left(t_{b}\right)\right\rangle$ already correspond to a specifications of the state of the particles at the time $t_{b}$ and denote by $N_{j} \in \Sigma_{t_{b}}^{t_{c}}$ the corresponding pattern of discharge of the counters. We can write

$$
\begin{aligned}
& \mathcal{F}\left(t_{c}, t_{b} ; N_{j}\right) \hat{\rho}^{\mathrm{T}}\left(t_{b}\right)= \\
& \quad=\rho_{j j}^{\mathrm{O}}\left(t_{b}\right) \mathcal{G}\left(t_{c}, t_{b}\right)\left\{\sum_{r s}\left|u_{j}, U_{r} ; t_{b}\right\rangle \rho_{r s}^{\mathrm{A}}\left(t_{b}\right)\left\langle u_{j}, U_{s} ; t_{b}\right|\right\} .
\end{aligned}
$$

from which, since $\mathcal{G}\left(t_{c}, t_{b}\right)$ is trace-preserving, it follows

$$
\begin{aligned}
& p_{j}\left(t_{b}\right) \equiv P\left(t_{c}, t_{b} ; N_{j}\right)=\operatorname{Tr}\left\{\mathcal{F}\left(t_{c}, t_{b} ; N_{j}\right) \hat{\rho}^{\mathrm{T}}\left(t_{b}\right)\right\}= \\
& =\rho_{j j}^{\mathrm{O}}\left(t_{b}\right) \operatorname{Tr}\left\{\sum_{r s}\left|u_{j}, U_{r} ; t_{b}\right\rangle \rho_{r s}^{\mathrm{A}}\left(t_{b}\right)\left\langle u_{j}, U_{s} ; t_{b}\right|\right\}=\rho_{j j}^{\mathrm{O}}\left(t_{b}\right),
\end{aligned}
$$

that is the prescription of usual elementary Quantum Theory, up to the correction introduced in (152) by the action of the mapping $\mathcal{G}\left(t_{b}, t_{a}\right)$.

\section{References}

1. A. Barchielli, L. Lanz and G. M. Prosperi, Nuovo Cimento, 72 B, 79 (1982); Foundation of Physics, 13, 779 (1983); Proceedings of the ISQM, p. 165, Tokyo 1984; G. M. Prosperi, Lect. Notes in Mathematics, Springer Verlag 1055, 301 (1984).

2. E. B. Davis, Quantum Theory of Open Systems, Academic Press, London, 1976.

3. G. Ludwig, Foundation of Quantum Mechanics, Springer, Berlin, 1982;. K. Kraus, States, effects and operation, Lecture Notes in Physycs, 90, Springer, Berlin, 1983. 
4. A. S. Holevo, Probabilistic and Statistical Aspects of Quantum Theory, North Holland, Amsterdam 1982; Statistical Structure of Quantum Theory, Springer, Berlin 2001.

5. T. Heinosaari and M. Ziman, The Mathematical Language of Quantum Theory, Cambridge University Press, Cambridge 20011; A. S. Holevo, Quantum Systems, Channels, Information. A Mathematical Introduction Series: De Gruyter Studies in Mathematical Physics 16.

6. A. Barchielli and M. Gregoratti, Quantum Trajectories and measurement in Continuous Time, Spriger Verlag, Berlin-Heidelberg 2009.

7. R. Grifths, J. Stat. Phys. 36, 219 (1984); ibid. 55, 11 (1987); Consistent Quantum Theory, Cambridge Unuiv. Press, 2002; M. Gell-Mann and J. B. Hartle, Proceedings of the ISQM, S. Kobayashi, H. Ezawa, Y. Murayama and S. Nomuda eds. Physical Society of Japan, Tokyo 1990; R. Omnes, J. Stat. Phys. 53, 893, 933, 957 (1988); ibid. 57, 357 (1989); ibid. 62(1991); Ann. Phys. N. Y., 201, 354 (1990); Rev. Mod. Phys. 64, 339 (1992).

8. B. d'Espagnat, Phys. Lett. A 124, 204 (1987); J. Stat. Phys. 56, 747 (1989); H. F. Dowker and A. Kent, Phys. Rev. Lett. 75, 3038 (1995); J. Stat. Phys. 82, 15575 (1996); G. Peruzzi and A. Rimini, Found. Phys. Lett. 11, 201 (1998);

9. H. D. Zeh, Found. Phys. 1, 69 (1970); E. Joos and H. D. Zeh, Z. Phys. B: Condensed Matter 59, 223 (1985); W. H. Zurek, Rev. Mod. Phys. 75, 715 (2003); B. Vacchini and K. Hornberger, Phys. Rep.478, 71 (2009).

10. A. Bassi and G. C. Ghirardi, Phys. Rep. 379, 257 (2003); A. Bassi, K. Lochan, S. Satin, T. P. Singh and H. Ulbricht, Rev. Mod. Phys. 85, 471 (2013).

11. G. C. Ghirardi, A. Rimini and T. Weber, Phys. Rev. D 34, 470 (1986); Phys. Rev. D 36, 3287 (1987); G. C. Ghirardi, R. Grassi and F. Benatti, Found. Phys. 25, 5 (1995); A. Bassi, G. C. Ghirardi, D. G. M. Salvetti, J. Phys. A: Math. Theory, 40, 13755 (2007).

12. P. Pearl, Phys. Rev. A 39, 2277 (1989); G. C. Ghirardi, P. Pearl and A. Rimini, Phys. Rev. A 42, 78 (1990).

13. S. L. Adler, Quantum Theory as a emergent phenomenon, Cambridge Univ. Press 2004.

14. L. Diosi and B. Lukacs, Annalen der Physik 44, 488 (1987); L. Diosi, Braz. J. Phys. 35, 260 (2005).

15. G. M. Prosperi, Int, Jour. Theor. Phys. 33, 118 (1994); AIP Conference Proceedings, 461, 91 (1999).

16. P. Caldirola, Nuovo Cimento A:45, 549; R. Bonifacio, AIP Conference proceeding, 461, 122 (1999), G. J. Milburn, Phys. Rev. A 44 R5401 (1991), E. Recami and R. H. A. Farias, Nuovo Cimento B /147, 765 (2009).

17. B. Misra and E. C. Sudarshan, J. Math. Phys. 18, 756 (1977).

18. V. Gorini, A. Kossakorowski and E. G. Sudarshan, J. Math. Phys. 17, 821 (1976); G. Lindblad, Comm. Math. Phys. 48, 119 (1976).

19. A. Barchielli and G. Lupieri, J. Math. Phys 26 ,2222 (1985).

20. E. S. Abers and B. W. Lee, Phys. Rep. 9, 1-141 (1073).

21. R. P, Feynman and A. R. Hibbs, Quantum Mechanics and Path Integrals, Mac CrawHill, New York, 1965. 